\title{
8 Infektionskrankheiten
}

\section{Gilles Wandeler, Petra Gastmeier, Kathrin Mühlemann}

Trotz bedeutender Fortschritte im Bereich der Prävention und der Therapie gehören Infektionen noch immer weltweit zu den wichtigsten Ursachen menschlicher Morbidität und Mortalität. Ein markantes Merkmal von Infektionskrankheiten ist ihre Übertragbarkeit, die je nach Übertragungsweg und Mitbeteiligung von lebenden Überträgern (Vektoren) auch stark durch Umweltfaktoren beeinflusst werden kann.

In diesem Kapitel geben wir eine Übersicht über die wesentlichen epidemiologischen Aspekte der Infektionskrankheiten, berücksichtigen dabei geografische Unterschiede und gehen in diesem Rahmen auch auf die Konzepte der Übertragungsdynamik ein. Wir konzentrieren uns dabei auf Infektionen und Konzepte, die aktuell von großer Bedeutung sind oder deren Bedeutung in Zukunft zunehmen wird.

Schweizerische Lernziele: CPH 49-58

\subsection{Allgemeine Konzepte}

\subsubsection{Merkmale einer Infektionskrankheit}

Infektionskrankheiten werden durch Krankheitserreger ausgelöst. Es handelt sich dabei um Mikroorganismen (Bakterien, Pilze, Protozoen, Würmer und Viren), die in den Körper eindringen und sich dort vermehren. Der Mensch dient diesen Mikroorganismen als Wirt. Infektionskrankheiten zeigen meist einen typischen zeitlichen Verlauf. Tab. 8.1 definiert die hierbei verwendeten Begriffe.

Tab. 8.1: Die wichtigsten Begriffe zum zeitlichen Ablauf einer Infektionskrankheit.

Ansteckung

Inkubationszeit

Krankheit

Asymptomatische Infektion
Kontakt, Etablierung und Vermehrung des Infektionserregers im Wirt

Zeitintervall zwischen der Ansteckung und dem Auftreten erster Symptome, z. B. durch erste Vermehrung des Erregers an der Eintrittspforte und anschließende Dissemination (Streuung) über die Blutbahn zum Zielorgan (s. Web-Abb. 8.1.1 auf unserer LehrbuchHomepage)

Zeitraum, der durch das Vorhandensein von Symptomen gekennzeichnet ist

Infektion, die bei einem Menschen keine Symptome verursacht Asymptomatische Infektionen können epidemiologisch wichtig sein, da Infizierte als Quellen für die Weiterverbreitung der Infektion in Frage kommen können. 
Ausscheidungsphase

Elimination

Immunität

Kolonisation

Latente Infektion
Zeitspanne, während der der Infektionserreger übertragbar ist Sie korreliert häufig mit der Krankheitsphase. Beispiel für wichtige Ausnahmen hiervon sind Hepatitis A-, Varizellen-, Influenza-, Parvovirus B19- und HIV-Infektionen.

Der Erreger wird durch das Abwehrsystem (und evtl. die Therapie) unschädlich gemacht.

Unempfindlichkeit gegenüber dem Erreger: Der Wirt kann nicht (mehr) angesteckt werden bzw. es kommt zu einer epidemiologisch bedeutungslosen abortiven Infektion, die zu keiner weiteren Erregerübertragung führt.

Vermehrung von Erregern (Bakterien, Pilze) auf Haut oder Schleimhäuten, ohne dass Krankheitssymptome auftreten

Die Kolonisation ist epidemiologisch wichtig, da hierdurch die Möglichkeit der Übertragung der Erreger auf andere Personen besteht.

Andauernde Infektion ohne Krankheitssymptome und evtl. auch ohne Vermehrung des Erregers im Wirt

Von epidemiologischer Bedeutung sind latente Infektionen aufgrund ihres Reaktivierungspotentials: Sie können unter ungünstigen Bedingungen wieder zu klinisch aktiven Infektionen werden.

Für die epidemiologische Beurteilung von Infektionskrankheiten ist es wichtig zu wissen, wie diese Stadien bei einer bestimmten Infektion verlaufen (Abb. 8.1). Auf unserer Lehrbuch-Homepage finden Sie hierzu noch in Web-Abb. 8.1.1 die Inkubationszeiten von wichtigen Infektionskrankheiten.

Epidemiologisch bedeutend sind die Stadien, während derer der Infektionserreger ausgeschieden wird und damit übertragen werden kann. Wie lange dieser Zeitraum ist, hängt von der Art des Erregers ab.

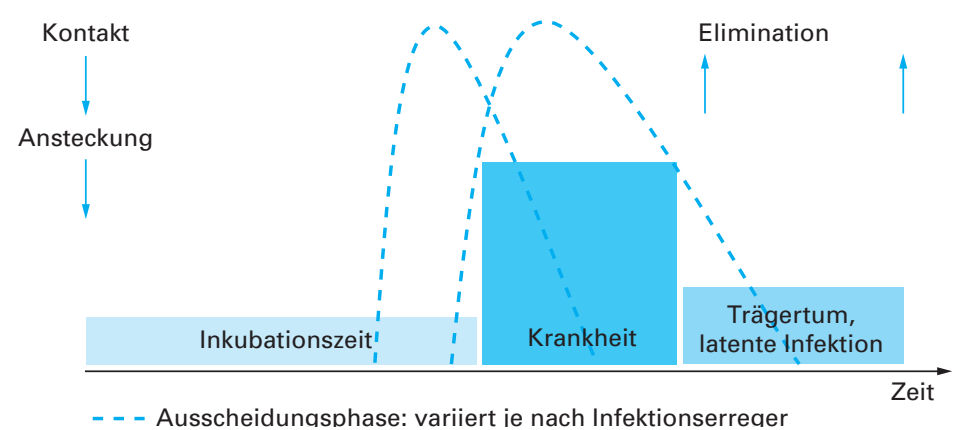

Abb. 8.1: Stadien einer Infektion. 


\subsection{2 Übertragungswege und Übertragungsdynamik}

\section{Übertragungswege}

Ein Schlüsselmerkmal von Infektionserregern ist die Übertragbarkeit von Wirt zu Wirt. Die möglichen Übertragungswege werden in Tab. 8.2 dargestellt. Als horizontale Übertragung bezeichnet man hierbei die Übertragung innerhalb einer Wirtspopulation, als vertikale Übertragung die Übertragung auf die nächste Generation, d. h. von der Mutter auf den Fetus oder das Neugeborene.

Tab. 8.2: Übertragungswege von Infektionserregern.

Übertragungsweg

Erreger, die auf diesem Weg übertragen werden

\section{Horizontale Übertragung}

\section{DIREKT}

Physischer Kontakt

Hände

Sexualkontakt

Aerogen

Tröpfchen

Aerosol

INDIREKT

Vehikel (unbelebt)

Gegenstände, Nahrung, Wasser

Blut u. a. biologische

Flüssigkeiten

Erde

Vektor (belebt)

Mücken, Zecken etc.

\section{Vertikale Übertragung}

Prä-, Perinatal
Viele multiresistente Bakterien (z. B. MRSA), Durchfallerreger, Respiratory syncytial Virus (RSV)

Erreger sexuell übertragbarer Infektionen [sexually transmitted infections (STI)]

Pneumokokken, Meningokokken, Gruppe A Streptokokken, respiratorische Viren (Influenza)

Tuberkelbakterien, Varizella-Zoster-Virus, Masernvirus

Multiresistente Keime, RSV

Salmonellen, Aspergillen,

HIV, Hepatitis-B-Viren, Hepatitis-C-Viren

Erreger von Tetanus und Gasbrand

Erreger von Malaria, Frühsommermeningoenzephalitis (FSME), Lyme Borreliose

Zytomegalievirus, Rötelnvirus, Toxoplasma gondii etc.

Eine Erläuterung hierzu: Die aerogene Übertragung geht in der Regel von den Sekreten der Atemwege aus.

(1) Tröpfchen: Während wir sprechen, husten, niesen, singen etc. werden Sekrettröpfchen mit einem Durchmesser von $\leq 5 \mathrm{~mm}$ in die Luft entlassen. Diese fallen aufgrund ihrer Größe nach 1-2 m Entfernung auf den Boden. Beim Einatmen gelangen sie nur bis in die oberen Atemwege. (2) Aerosol: Durch das Verdampfen der Tröpfchen entstehen Tröpfchenkerne (Durchmesser $<5 \mu \mathrm{m}$ ), welche aufgrund ihrer geringen Größe schweben und über weite Distanzen übertragen werden können. Beim Einatmen gelangen Aerosole bis in die unteren Atemwege. Nur wenige Infektionserreger können im geringen Feuchtigkeitsgehalt eines Aerosols überleben. 
Genaue Kenntnis über den Übertragungsweg eines Infektionserregers ist einer der Grundpfeiler für die Expositionsprophylaxe (s. Kapitel 8.4.3). Der Übertragungsweg für einen bestimmten Infektionserreger wird in der Regel aus den epidemiologischen Daten abgeleitet. Nur selten wurden Übertragungswege im Tierexperiment oder in klinischen Studien bewiesen.

\section{Übertragungsdynamik}

Die Übertragungsdynamik beschreibt die Geschwindigkeit und das Muster der Ausbreitung eines Infektionserregers in einer Population. Sie wird heute oft in mathematischen Modellen beschrieben. Wichtige Begriffe und Merkmale hierzu werden in Tab. 8.3 definiert. Auf unserer Lehrbuch-Homepage zeigt Web-Abb. 8.1.2 den zeitlichen Verlauf der effektiven Reproduktionsrate (R) einer Infektion innerhalb einer voll empfänglichen Population und einer Population, in der $30 \%$ der Personen gegen die Erkrankung immun sind.

\subsubsection{Epidemie}

\section{Definition}

Eine Epidemie wird definiert als eine Zunahme an neuen Erkrankungsfällen über eine zu erwartende Basisrate hinaus innerhalb eines definierten Zeitraums in einer definierten Region. Es handelt sich also um eine zeitliche und örtliche Häufung einer (Infektions-) Krankheit innerhalb einer Population. Dies steht im Gegensatz zur endemischen Situation, in welcher eine Infektion in einer definierten Population mit stabiler Rate präsent ist. Betrifft eine Epidemie mehrere Kontinente, so spricht man von einer Pandemie. Bekannte Beispiele für eine Pandemie sind die Influenza-Pandemie von 1918 1919 und die sich seit dem Ende des 20. Jh. weltweit ausbreitende HIV-Pandemie.

Bei systematisch überwachten Infektionskrankheiten, wie z. B. bei der Influenza, ist die Basisrate gut bekannt, sodass für wiederkehrende (saisonale) Epidemien ein Schwellenwert definiert wird. Dieser Schwellenwert beträgt für die Influenza 50 Fälle von „Influenza-like Syndrome“ pro 100.000 Einwohner. Bei nicht systematisch überwachten Infektionskrankheiten oder kleinen, lokal begrenzten Epidemien führt oft der subjektive Eindruck einzelner Beobachter zur Entdeckung einer Epidemie.

In der Schweiz und in Deutschland sind Häufungen von Infektionskrankheiten meldepflichtig (> 2 unerwartete oder bedrohliche Fälle vom gleichen Ort, auch wenn der Erreger nicht meldepflichtig ist; s. Kap. 8.2.3).

\section{Begriffe}

Grundsätzlich kann jeder Infektionserreger zu einer Epidemie führen. Hierzu müssen jedoch einige Bedingungen erfüllt sein. So muss eine Quelle für den Infektionserreger existieren, der Infektionserreger muss ein gewisses Maß an Kontagiosität aufweisen, und es müssen genügend empfängliche Individuen zur Verfügung stehen, damit die Reproduktionsrate der Infektion $>1$ ist (s. Kap. 8.1.2). Entwicklungsstand, Einkommen und lokale sozio-kulturelle Eigenschaften einer Bevölkerung sowie die Schwächen der Gesundheitssysteme können daher ebenso wie Umwelt und Klima bei der Entwicklung einer Epidemie eine wichtige Rolle spielen. 
Tab. 8.3: Die wichtigsten Begriffe zur Übertragungsdynamik von Infektionserregern in einer Population.

\section{Kontagiosität}

(Ansteckbarkeit)

Populationsdichte

Reproduktionsrate

Basale $\mathbf{R}_{\mathbf{0}}$

Effektive R

"Superspreader"

Herdenimmunität Population
Maß für die Wahrscheinlichkeit, dass die Übertragung eines Erregers stattfindet. Sie ist abhängig vom Übertragungsweg (s. Tab. 8.1.2) sowie den biologischen Merkmalen des Infektionserregers (z. B. Adhärenzfaktoren) und des Wirtes (z. B. Rezeptoren). Anzahl der Personen pro Fläche $\left(\mathrm{km}^{2}\right)$. Enges Zusammenleben auf kleinem Raum wird auch als „Crowding" bezeichnet. Die Populationsdichte beeinflusst die Geschwindigkeit, mit der sich ein Infektionserreger ausbreiten kann. Sie korreliert in der Regel invers mit dem Wohlstand einer Population.

Durchmischung einer Menschliche Populationen mischen sich in der Regel nicht homogen, sie gruppieren sich z. B. nach Interessensgemeinschaft, sozialer Schichtung, Verhalten etc. Dies kann auch das Übertragungsmuster einer Infektionskrankheit beeinflussen. Beispiel: Sexuell-übertragene Infektionen bei Personen mit besonderem Sexualverhalten, Masernausbruch in Gemeinschaften von Impfgegnern

Die Reproduktionsrate beschreibt hier die Anzahl an neuen Infektionen, die von einem Fall ausgehen (s. Abb. 8.3).

Die basale Reproduktionsrate $\left(R_{0}\right)$ bezieht sich auf eine Population, in der alle Mitglieder für die Infektion empfänglich sind. Sie wird durch die Kontagiosität, die Populationsdichte und die Durchmischung einer Population bestimmt. In verschiedenen Populationen kann sie deshalb für denselben Erreger unterschiedliche Werte annehmen.

Beispiele für $R_{0}$

Masern $\quad$ 5-18

Keuchhusten (Pertussis) 10-18

Windpocken (Varizellen) $\quad 7-14$

HIV 2-12

Die effektive Reproduktionsrate berechnet man für eine Population, in der nicht alle Mitglieder für die Infektion empfänglich sind. $R \leq R_{0}$

Wenn $R>1$, dann nimmt die Anzahl der Infektionsfälle zu.

Wenn $R=1$, dann bleibt die Anzahl der Infektionsfälle konstant.

Wenn $R<1$, dann nimmt die Anzahl der Infektionsfälle ab.

Im Verlauf einer Epidemie (s. Kap. 8.1.3) ist R zu Beginn > 1. Je mehr Personen der exponierten Population die Infektion durchlebt haben, desto stärker sinkt die Anzahl der für die Infektion empfänglichen Personen. Ist dann der kritische Punkt erreicht, bei dem nur noch wenige empfängliche Personen zur Verfügung stehen, sinkt $R<1$ und die Epidemie kommt zum Stillstand.

Einige Individuen zeichnen sich durch ein überdurchschnittlich hohes $R_{0}$ bzw. $R$ aus. Dabei spielen bislang unbekannte biologische Merkmale und/oder ein besonderes Verhalten eine Rolle. So war z. B. im Februar 2002 ein einziger Patient in Hongkong für den Beginn der SARS-Epidemie verantwortlich, indem er in einem Hotel mindestens 10 Personen ansteckte (durchschnittliche $\mathrm{R}_{0}$ für SARS: 2-3).

Als Herdenimmunität (Herd Immunity) bezeichnet man einen Effekt innerhalb einer Population (der "Herde"), der dann entsteht, wenn dort eine durch Impfung erzeugte oder durch Infektion erworbene Immunität gegen einen Krankheitserreger so weit verbreitet ist, dass in der Population auch nicht-immune Personen geschützt sind, da der Erreger sich nicht weiter ausbreiten kann. 
Wichtige Begriffe im Rahmen einer Epidemie sind:

- Inkubationszeit (Kap. 8.1.1): Sie kann einen wichtigen Hinweis auf den (noch unbekannten) Infektionserreger einer Epidemie geben.

- Attackrate (Kontagionsindex): Sie beschreibt das Verhältnis von neu infizierten Personen bezogen auf alle exponierten Personen. Durch sie lassen sich Hinweise auf die Kontagiosität und damit die Identität des Infektionserregers gewinnen.

- Basale Reproduktionsrate (s. Kap. 8.1.2)

- Epidemiekurve: Sie zeigt den zeitlichen Verlauf der Anzahl an Fällen während einer Epidemie in Form einer graphischen Darstellung. Ein typisches Beispiel ist die Darstellung der saisonalen Influenzaepidemie (s. Abb. 8.2).

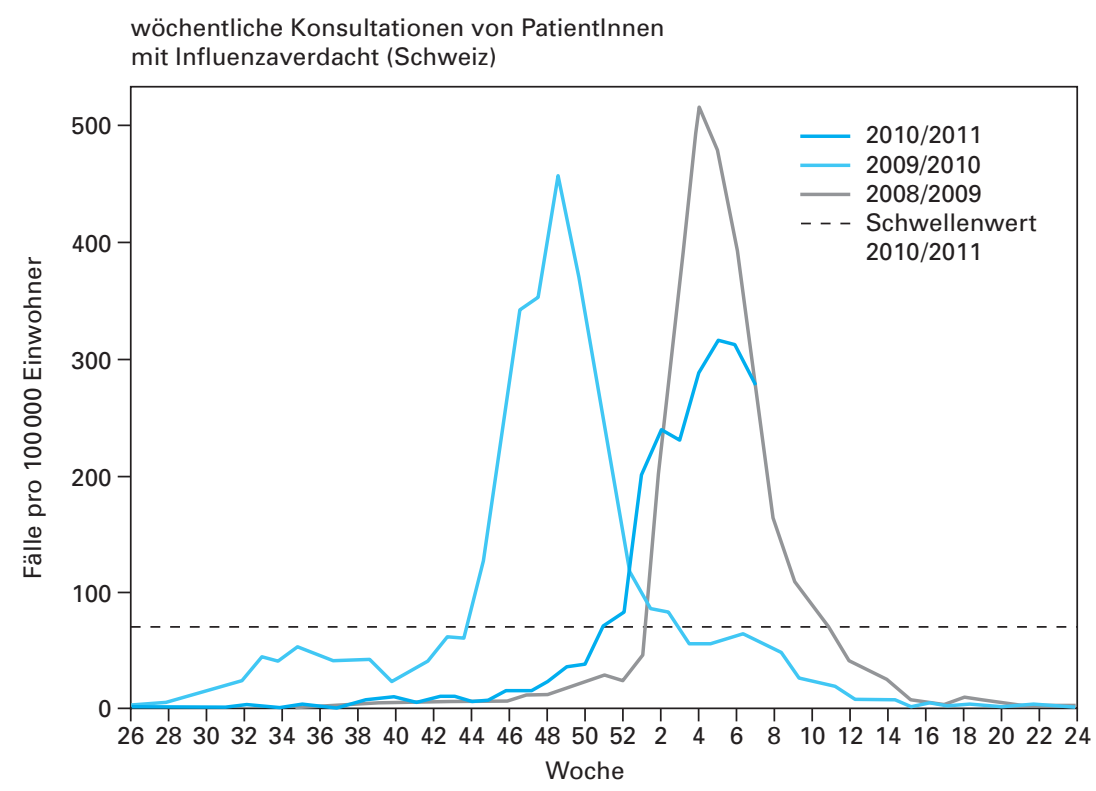

Abb. 8.2: Epidemiekurven der saisonalen Influenzaepidemien in der Schweiz in den Zeiträumen 2008/2009, 2009/2010 und 2010/2011. Dargestellt ist die Zahl der wöchentlichen ärztlichen Konsultationen von Patienten mit Influenzaverdacht. Eingezeichnet ist darüber hinaus der errechnete saisonale Influenza-Schwellenwert für den Zeitraum 2010/2011 (Quelle: Bundesamt für Gesundheit BAG).

Die Form der Epidemiekurve kann einen Hinweis auf die Art der Quelle und den Übertragungsweg geben. Zudem kann in manchen Fällen ("Common source" mit Punktquelle, s.u.) aus der Epidemiekurve die Inkubationszeit des Infektionserregers errechnet werden, was dann wiederum Rückschlüsse auf die Art des Infektionserregers erlaubt (s. Abb. 8.3). Eine Epidemie, die von einer spezifischen Infektionsquelle ausgeht und nicht zusätzlich von Person zu Person übertragen wird, wird als Common-sourceEpidemie bezeichnet. Sie kann entweder durch eine einmalige oder auch durch wiederholte Exposition hervorgerufen werden. Bei einer einmaligen Exposition spricht man 

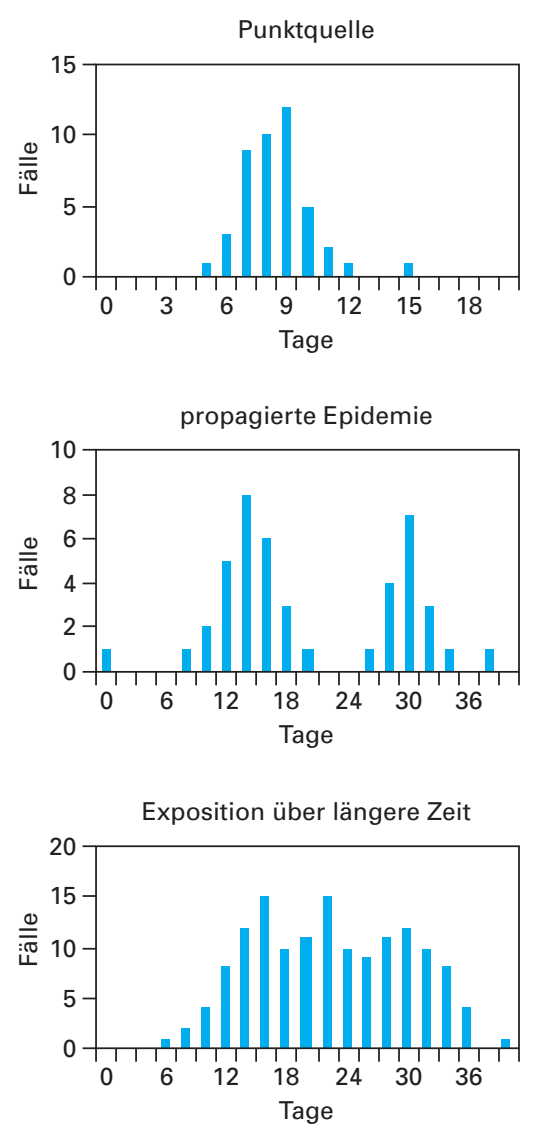

\section{Common source-Epidemie mit Punktquelle}

Beispiel: Kartoffelsalat bei einem Büffet.

Die Zeit zwischen der Exposition (Tag 0 = Büffet) und dem Gipfel der Epidemie (Tag 9) entspricht der durchschnittlichen Inkubationszeit.

\section{Propagierte Epidemie}

Beispiel: Kinderkrankheiten wie Röteln, Masern Ein Indexfall (Tag 0, erster Fall und Ausgangspunkt der Epidemie) führt zu einer ersten Welle von Fällen. Diese stecken weitere Personen an, was zu einer zweiten (und evtl. dritten Welle etc.) führt. Ein solches Bild wird bei Infektionskrankheiten beobachtet, die direkt von Person zu Person übertragen werden und hoch kontagiös sind. Die Inkubationszeit ergibt sich aus der Zeitdifferenz zwischen dem Indexfall und dem Gipfel der ersten Welle bzw. zwischen den Gipfeln zweier Wellen.

\section{Common source-Epidemie mit verlängerter Exposition} Beispiele: kontaminierte Wasserquelle Ist eine Ansteckungsquelle über längere Zeit aktiv, so ergibt sich eine langgestreckte Kurve. Jedoch kann bei bekanntem Erreger die aus der Literatur ersichtliche durchschnittliche Inkubationszeit bei der Identifikation der Quelle behilflich sein. Die Quelle musste zur Zeit des ersten Falles abzüglich der kürzesten Inkubationszeit bereits aktiv gewesen sein.

Abb. 8.3: Beispiele verschiedener Epidemiekurven (Quelle: Bundesamt für Gesundheit BAG).

von einer Point-source-Epidemie (Epidemie mit Punktquelle), bei wiederholter Exposition von Extended epidemic (Epidemie mit verlängerter Exposition). In beiden Fällen lässt sich die "Attackrate" (s.o.) berechnen. Falls Infektionen zu verschiedenen Zeitpunkten auftreten und von Person zu Person übertragen werden, spricht man von Propagated-source-Epidemien (propagierte Epidemie; Beispiel: Masern-Epidemie).

\section{Epidemieabklärung}

Damit die Ausbreitung einer Epidemie möglichst früh in ihrem Verlauf gestoppt oder eingeschränkt werden kann, müssen Infektionserreger, Quelle und Übertragungsweg ermittelt werden. Basierend auf diesen Kenntnissen werden dann Kontrollstrategien formuliert und umgesetzt. Ein weiterer wichtiger Punkt ist die laufende und angemessene Information der Öffentlichkeit. Die Web-Tab. 8.1.1 auf unserer Lehrbuch-Homepage zeigt die von den Centers for Disease Control and Prevention (CDC, Atlanta, USA) entwickelten und empfohlenen allgemeinen Schritte im Rahmen einer Epidemie-Abklärung. Im individuellen Fall werden diese Schritte nicht immer präzise entsprechend 
dieser Reihefolge ausgeführt. Je nach Situation ist ein paralleles Vorgehen angezeigt, oder es müssen Schritte wiederholt werden.

Die Zuständigkeit für eine Epidemieabklärung liegt grundsätzlich bei den Gesundheitsbehörden und damit in der Hand des Staates und seiner Organe. Dies ist wichtig, da unter Umständen beachtliche finanzielle und gesundheitsschädigende Folgen aus einer Epidemie entstehen können. Darüber hinaus sind im Rahmen der Kontrollstrategien eventuell notwendige Verbote und andere freiheitseinschränkende Maßnahmen zu treffen. Die Behörden können die Aufgabe einer Epidemieabklärung aber auch delegieren. So wird die Abklärung einer nosokomialen Epidemie (d. h. einer Epidemie, die von einem Krankenhaus oder einer Pflegeeinrichtung ausgeht, s. Kap. 8.3.2) in der Regel durch die lokalen Verantwortlichen für Infektionskontrolle durchgeführt. In Deutschland ist das Robert Koch-Institut (RKI) die zentrale Einrichtung der Bundesregierung auf dem Gebiet der Krankheitsüberwachung und -prävention. Die Zuständigkeit für den Infektionsschutz liegt jedoch bei den einzelnen Bundesländern. Daher kann das RKI z. B. für den Epidemie-Fall nur mobile Teams bereithalten, die vor Ort auf Einladung der Länder unterstützend tätig sein können.

Einige Infektionserreger verursachen regelmäßig wiederkehrende Epidemien. Typische Beispiele hierfür sind die saisonalen Epidemien durch respiratorische Viren (Influenzaviren, Respiratory Syncytial Virus etc.). Die Regelmäßigkeit dieser Epidemien erlaubt Voraussagen für den weiteren Verlauf und somit die rechtzeitige Einleitung von Kontrollstrategien (Beispiel: jährliche Impfung gegen Influenza).

\section{Internet-Ressourcen}

Auf unserer Lehrbuch-Homepage (www.public-health-kompakt.de) finden Sie Hinweise auf weiterführende Literatur, zusätzliche Abbildungen und Tabellen sowie Links zu den erwähnten Institutionen (z. B. zu den Centers for Disease Control and Prevention in den USA).

\section{2 Überwachung}

\subsubsection{Ziele der Überwachung}

Überwachung oder Surveillance definieren die amerikanischen Centers for Disease Contro and Prevention/ (CDC, Atlanta, USA) als "kontinuierliche, systematische Erfassung und Interpretation von Gesundheitsdaten, die für die Planung, Implementierung und Evaluation von Public-Health-Maßnahmen unerlässlich sind". Das Hauptziel eines solchen Überwachungssystems ist es, systematische Veränderungen der Neuerkrankungsraten (Inzidenzen) bei bestimmten Krankheiten zu erkennen, um dann adäquate Kontrollstrategien einzuleiten. Besonders wichtig ist zudem die frühzeitige Veröffentlichung der erhobenen und analysierten Daten.

\subsubsection{Gesetzliche Grundlagen und Rahmenbedingungen}

Die weltweite Überwachung von Infektionskrankheiten geschieht über spezifische nationale und internationale Melde- und Informationssysteme. Die passenden Links zu den entsprechenden Internetseiten finden Sie auf unserer Lehrbuch-Homepage. 


\section{Robert Koch-Institut (RKI)}

In Deutschland ist das Robert-Koch-Institut die zentrale Einrichtung des Bundes auf dem Gebiet der Krankheitsüberwachung und -prävention. Es ist damit auch ein wichtiges Zentrum der anwendungs- und maßnahmenorientierten biomedizinischen Forschung. Seine Kernaufgaben sind die Erkennung, Verhütung und Bekämpfung von Krankheiten, wobei der Schwerpunkt auf den Infektionskrankheiten liegt. Das RKI berät die zuständigen Bundesministerien, insbesondere das Bundesministerium für Gesundheit (BMG) und unterstützt die entsprechenden Institutionen bei der Entwicklung von Normen und Standards in diesem Bereich. Es informiert und berät die Fachleute ebenso wie die breite Öffentlichkeit. Im Hinblick auf das Erkennen von gesundheitlichen Gefährdungen und Risiken nimmt das RKI eine zentrale "Antennenfunktion" im Sinne eines Frühwarnsystems wahr.

\section{Bundesamt fürr Gesundheit (BAG)}

In der Schweiz ist die Bekämpfung übertragbarer Krankheiten, die eine Gefährdung der öffentlichen Gesundheit darstellen, Aufgabe der Abteilung Übertragbare Krankheiten des Bundesamts für Gesundheit. Das BAG arbeitet dabei eng mit den Kantonen, den internationalen Gesundheitsbehörden und weiteren Partnern zusammen. Im Rahmen dieser Aufgabe überwacht es das Auftreten übertragbarer Krankheiten, legt Präventionsund Kontrollstrategien fest, erlässt Weisungen, bereitet Verordnungen und Gesetze vor, erarbeitet Empfehlungen für die Ärzteschaft und die Bevölkerung und publiziert regelmäßig Berichte zur aktuellen epidemiologischen Situation.

\section{World Health Organization (WHO)}

Bei der internationalen Überwachung spielt die WHO eine zentrale Rolle. Sie leitet globale wissenschaftliche Netzwerke, wie das Global Influenza Surveillance Network. Die dort erhobenen Daten zu den aktuell zirkulierenden Influenzaviren-Subtypen werden beispielsweise als Grundlage für die Empfehlung der Zusammensetzung des Influenzaimpfstoffes der nächsten Saison benötigt.

\section{International Health Regulations (IHR)}

Die gesetzliche Basis für die internationale Autorität der WHO im Rahmen der Epidemie-Kontrolle bilden die International Health Regulations (Internationale Gesundheitsvorschriften). Unter diesem Namen wurde 1969 erstmals ein Dokument veröffentlicht, das die obligatorische Meldung der drei wichtigen übertragbaren Krankheiten Cholera, Gelbfieber und Pest empfahl. Es verblieb über Jahre unverändert. Die lang erwartete Revision der IHR wurde u. a. durch die zunehmende Globalisierung (Handel, Tourismus) sowie durch das Auftreten damit zusammenhängender, neuer Bedrohungen für die öffentliche Gesundheit (u.a. die SARS-Pandemie) nötig. Die Arbeiten hieran konnten 2005 abgeschlossen werden. Die neuen IHR wurden anschließend von 194 Ländern unterzeichnet und sind seit 2007 völkerrechtlich bindend. Die WHO hat nun die Möglichkeit, Vorgaben hinsichtlich der Überwachung und der Kontrolle von Ereignissen von internationaler Tragweite zu machen, die die öffentliche Gesundheit bedrohen. 
Diese müssen dann von den Mitgliedstaaten umgesetzt werden. Der Schwerpunkt der neuen IHR liegt jedoch vor allem in der Standardisierung der Meldungen von Public Health-Bedrohungen und -Notfällen.

\section{Global Outbreak Alert and Response Network (GOARN)}

Um die globalen Antworten auf neue Epidemien (emerging infections) schnell und effizient unterstützen und koordinieren zu können, gründete die WHO im Jahr 2000 das Global Outbreak Alert and Response Network. GOARN beruht auf der Zusammenarbeit von Hunderten internationaler, interdisziplinärer Teams, welche im Falle einer neuen Epidemie von möglicher globaler Bedeutung ihre Unterstützung anbieten.

\section{European Centre for Disease Prevention and Control (ECDC)}

Länderübergreifende europäische Daten werden zudem durch das ECDC publiziert.

\section{Nationale Meldesysteme}

In vielen Ländern besteht eine gesetzliche Meldepflicht für definierte übertragbare Krankheiten. Gesetzliche Grundlagen für die Meldesysteme sind in Deutschland das Infektionsschutzgesetz und in der Schweiz das Epidemiengesetz.

\subsubsection{Methodik und Meldesysteme}

Das Sammeln von Daten über Infektionserreger und Infektionskrankheiten kann grundsätzlich durch aktive oder passive Überwachung geschehen. Die passive Überwachung beruht auf der Analyse von Daten, welche routinemäßig (und mit unterschiedlicher Systematik) erhoben werden. Bei der aktiven Überwachung werden hingegen Daten erhoben, die im täglichen Routinebetrieb nicht systematisch gesammelt werden. Diese Art der Überwachung ist genauer und weniger anfällig für systematische Verzerrungen (Meldebias). Allerdings ist sie aufwändiger und wird deshalb gezielter eingesetzt als die passive Überwachung.

Je nach Land werden zusätzlich zu den WHO-meldepflichtigen Erkrankungen bis zu 50 weitere Infektionskrankheiten überwacht. Die schematische Darstellung in Abb. 8.4 verdeutlicht den Meldeablauf, der beim Auftreten von meldepflichtigen Erkrankungen in der Schweiz eingehalten werden muss. Meldende Stellen sind die diagnostizierenden ÄrztInnen sowie die Laboratorien. Aufgrund des föderalistischen Schweizer Systems erfolgt die Meldung zuerst an das zuständige Kantonsarztamt, welches die Information an das Bundesamt für Gesundheit (BAG) weiterleitet.

Eine Beschreibung des Meldesystems in Deutschland findet sich im Gesetz zur Verhütung und Bekämpfung von Infektionskrankheiten beim Menschen im 3. Abschnitt Meldewesen (s. Internetquellen auf unserer Lehrbuch-Homepage). Dort sehen Sie auch die Web-Abb. 8.2.1., die als Beispiel den Daten- und Informationsfluss vom und zum Robert Koch-Institut während der EHEC/HUS-Epidemie ${ }^{25}$ in Deutschland im Frühjahr 2011 zeigt.

25 EHEC = enterohämorrhagische Escherichia coli; HUS = hämolytisch-urämisches Syndrom 


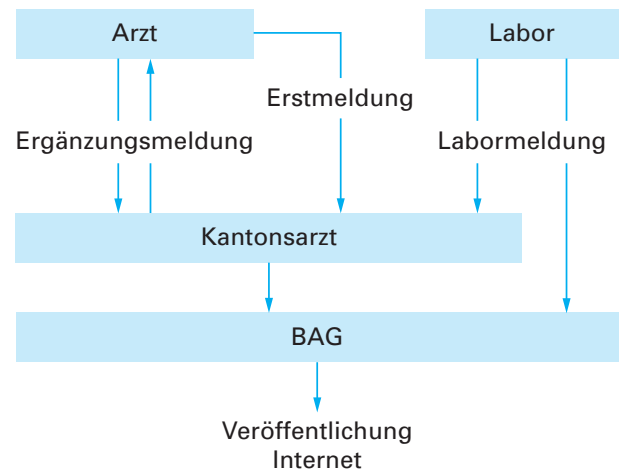

Abb. 8.4: Meldeablauf bei einer meldepflichtigen Infektionskrankheit in der Schweiz. Die Daten laufen beim Bundesamt für Gesundheit (BAG) zusammen, wo sie erfasst, analysiert und schließlich veröffentlicht werden. (Quelle: modifiziert nach Bundesamt für Gesundheit (BAG), Schweiz).

Meldepflichtige Infektionen müssen in einem festgelegten Zeitintervall in vorgegebener Form an die zuständige Stelle gemeldet werden. Die Dringlichkeit einer Meldung richtet sich dabei nach dem Risikopotential der Erkrankung und der Notwendigkeit bzw. Möglichkeit, unverzüglich Kontrollmaßnahmen zu ergreifen. In der Schweiz müssen beispielsweise Infektionskrankheiten wie Anthrax, Diphtherie, SARS und das virale hämorrhagische Fieber innerhalb von 24 Stunden gemeldet werden. Für andere Erkrankungen wie HIV/AIDS, Malaria oder Tuberkulose ist eine Meldung innerhalb von einer Woche vorgegeben (die vollständigen Listen finden sie auf der Homepage des BAG). Zur Meldung werden definierte Meldeformulare benutzt. Bei einigen Infektionskrankheiten erfolgt die Meldung anonym (Beispiel: HIV). Für bestimmte Infektionserreger (z. B. invasive Pneumokokkeninfektion, Tuberkulose) ist nach der Erstmeldung eine Ergänzungsmeldung mit Hilfe eines von den Behörden zugestellten Fragebogens durchzuführen.

Nicht alle Infektionen, die von epidemiologischer Bedeutung sind, sind auch meldepflichtig. Hierfür gibt es verschiedene Gründe. So ist z. B. die umfassende Meldung aller Fälle bei sehr häufigen Infektionskrankheiten, wie etwa Influenza, nicht zweckmäßig. Hier genügt es, über ein so genanntes Sentinella-System [sentinel system; von sentinel (engl.): Wächter] die Daten zur Inzidenz der Erkrankung in Form einer repräsentativen Stichprobe zu erheben. Die Teilnahme an einem solchen Sentinella-System ist in der Regel freiwillig.

In der Schweiz wurde das Sentinella-Meldesystem 1986 ins Leben gerufen. Dem Netzwerk gehören zwischen 150 und 250 ÄrztInnen an, die in der Grundversorgung tätig sind. Dies entspricht etwa 3\% der Allgemeinmediziner, Internisten und Pädiater in der Schweiz. Die Anfrage zur Teilnahme an diesem System geschieht nach statistischen Prinzipien, sodass die teilnehmenden ÄrztInnen eine repräsentative Auswahl darstellen. Die Teilnehmerlnnen am Sentinella-Meldesystem senden wöchentlich Daten zu ausgewählten Themen an das Bundesamt für Gesundheit. Klassische Beispiele für Erkrankungen, die über dieses System erfasst werden, sind neben der Virusgrippe (Influenza) auch andere Infektionskrankheiten, die sich durch Impfung verhüten lassen, wie Masern und Mumps. In den letzten Jahren hat sich das Spektrum der über das Sentinella-Netzwerk erhobenen Erkrankungen auch auf nicht-infektiöse Krankheiten (z. B. Suizidversuche, Antibiotikaverschreibung, Depression) ausgedehnt. 
Seit 1995 existiert in der Schweiz darüber hinaus auch ein Netzwerk zur Erfassung von wichtigen, aber seltenen pädiatrischen Infektionskrankheiten, die Swiss Paediatric Surveillance Unit (SPSU). Überwachte Themen sind hier z. B. die "akute schlaffe Lähmung", und das "konnatale Rötelnsyndrom“. Antibiotikaresistenzen werden in der Schweiz seit 2007 durch das nationale Überwachungssystem ANRESIS registriert (den passenden Link finden Sie auf unserer Lehrbuch-Homepage). Auch dieses System beruht auf dem freiwilligen Sentinella-Prinzip. Hier werden Routinedaten von statistisch ausgewählten Mikrobiologielabors gesammelt.

In Deutschland wurde mit der Antibiotika-Resistenz-Surveillance (ARS) ein repräsentatives, flächendeckendes System zur Überwachung von Antibiotika-Resistenzen eingerichtet, das sowohl die stationäre Krankenversorgung als auch den Sektor der ambulanten Versorgung einbezieht. Auf diese Weise werden aussagekräftige Daten zur Epidemiologie der Antibiotika-Resistenz in Deutschland gewonnen. Insbesondere können die Daten nun auch differenziert im Hinblick auf bestimmte Strukturmerkmale der Krankenversorgung und auf die regionale Verteilung betrachtet werden. ARS wurde als laborgestütztes Surveillancesystem zur kontinuierlichen Erhebung von Daten konzipiert, das Daten zum gesamten Spektrum klinisch relevanter bakterieller Erreger aus dem Routinebetrieb der Krankenversorgung sammelt. Projektteilnehmer und damit Datenlieferanten sind Laboratorien, die Proben aus medizinischen Versorgungseinrichtungen und Arztpraxen mikrobiologisch untersuchen.

\section{Internet-Ressourcen}

Auf unserer Lehrbuch-Homepage (www.public-health-kompakt.de) finden Sie Hinweise auf weiterführende Literatur und Links zu den erwähnten Institutionen.

- In der Schweiz werden die gesammelten Überwachungsdaten auf der Webseite des Bundesamts für Gesundheit veröffentlicht und zudem im wöchentlichen Bulletin des BAG publiziert.

- In Deutschland werden die Daten der Öffentlichkeit vom Robert Koch-Institut zur Verfügung gestellt.

\subsection{Epidemiologie wichtiger Infektionskrankheiten}

\subsubsection{Mortalität und Morbidität infolge von Infektionskrankheiten}

Weltweit verursachen Infektionskrankheiten etwa $25 \%$ aller Todesfälle (vgl. Kap. 9.1). Vier der zehn häufigsten Todesursachen sind Infektionen. Die relative Verteilung der zehn wichtigsten Todesursachen weltweit variiert jedoch in Abhängigkeit von der geografischen Lage und dem Lebensstandard der Menschen. So sterben in Low incomeLändern jährlich über 1 Mio. Menschen (11\% der Todesfälle) an Infektionen der unteren Luftwege und 760.000 Personen (8\%) an Durchfallerkrankungen. In High-income-Ländern, wo ischämische kardiovaskuläre Erkrankungen (s. Kap. 7.1) und Lungenkrebs (s. Kap. 7.2) als Todesursachen im Vordergrund stehen, sind Pneumonien nur für $4 \%$ der Todesfälle verantwortlich. Pneumonien und Durchfallerkrankungen sind global gesehen auch die wichtigsten Gründe für Morbidität. Die hierdurch hervorgerufene geschätzte Krankheitslast beträgt $11 \%$ der weltweit verlorenen gesunden Le- 
bensjahre, ausgedrückt DALYs (s. Web-Tab. 9.1.1 auf unserer Lehrbuch-Homepage). HIV/AIDS und Tuberkulose gehören ebenfalls zu den häufigsten Todesursachen. Besonders betroffen ist hier die Subsahara-Region in Afrika. Dort hat die HIV/AIDS-Pandemie in den letzten 20 Jahren zu einer massiven Senkung der Lebenserwartung geführt.

Die Mehrheit der Todesfälle durch Infektionskrankheiten bei Kindern ereignet sich in der Gruppe der unter 5-Jährigen in Entwicklungsländern. In dieser Altersgruppe haben akute respiratorische Infektionen und Durchfallerkrankungen den größten Einfluss auf die Gesamtmorbidität und -mortalität (s. Tab. 9.3). Zusammen mit Geburtskomplikationen und Malariainfektionen tragen diese Infektionen zu drei Viertel der Todesfälle bei. So sind z. B. über $40 \%$ der an einer Pneumonie verstorbenen Patienten Kinder unter 5 Jahren, hiervon leben $95 \%$ in den so genannten Entwicklungsländern.

\subsubsection{Global bedeutende Infektionskrankheiten am Beispiel von Malaria und HIV/AIDS}

\section{Malaria}

Die Malaria gehört zu den häufigsten Infektionskrankheiten weltweit. Jährlich werden über 200 Mio. Menschen infiziert, über 800.000 Personen sterben daran pro Jahr. Letzteres sind zu $80 \%$ Kinder aus Subsahara-Afrika. Der Erreger ist ein Parasit, der durch Anopheles-Mücken übertragen wird (s. Kap. 8.3.4). Diese Mücken sind in den meisten tropischen Regionen endemisch. Die höchste Morbidität und Mortalität ruft Plasmodium falciparum hervor, das für $90 \%$ der Malariainfektionen weltweit verantwortlich ist. Der zweithäufigste Malaria-Erreger ist Plasmodium vivax, der vor allem in Asien, Südamerika und im Westpazifik vorkommt. Infektionen durch diesen Erreger zeigen in der Regel einen gutartigen klinischen Verlauf. Seltenere Malaria-Erreger sind Plasmodium ovale, Plasmodium malariae und Plasmodium knowlesi.

Die wichtigen Pfeiler der Malariaprävention sind

- die Expositionsprophylaxe (s.a. Kap. 8.4.3) durch Mückenschutz, wie z. B. deckende Kleidung, topische Repellentien (Abwehrstoffe, Vergrämungsmittel) und mit Insektizid imprägnierte Moskitonetze

- die Chemoprophylaxe gegen Malaria in Form einer medikamentösen Dauerprophylaxe

Abb. 8.5 gibt einen Überblick über die aktuelle Verbreitung der Malaria sowie über die empfohlenen Möglichkeiten der Chemoprophylaxe. Für Aufenthalte in vielen afrikanischen Ländern, in Papua-Neuguinea und einem Teil von Indonesien wird eine Prophylaxe mit Mefloquin, Atovaquon/Proguanil oder Doxycyclin empfohlen. In anderen Gebieten mit niedriger Malariaverbreitung wird eine Selbstmedikation als Notfalltherapie bei Auftreten von klassischen Symptomen wie hohes Fieber, Kopf- und Gliederschmerzen empfohlen. Reisende, die eine Malaria entwickeln, haben häufig die Empfehlungen zur Expositions- und Chemoprophylaxe nicht befolgt. Dieses Risiko ist besonders hoch bei MigrantInnen, die ihre einheimischen Verwandten in tropischen Ländern besuchen (Visiting Friends and Relatives).

Malaria ist eine wichtige Ursache für unklare Fieberzustände (Status febrilis) nach der Rückkehr aus den Tropen. Die ersten Symptome treten klassischerweise zwischen einer und vier Wochen nach Exposition auf. Die Wahrscheinlichkeit einer Plasmodium falci- 


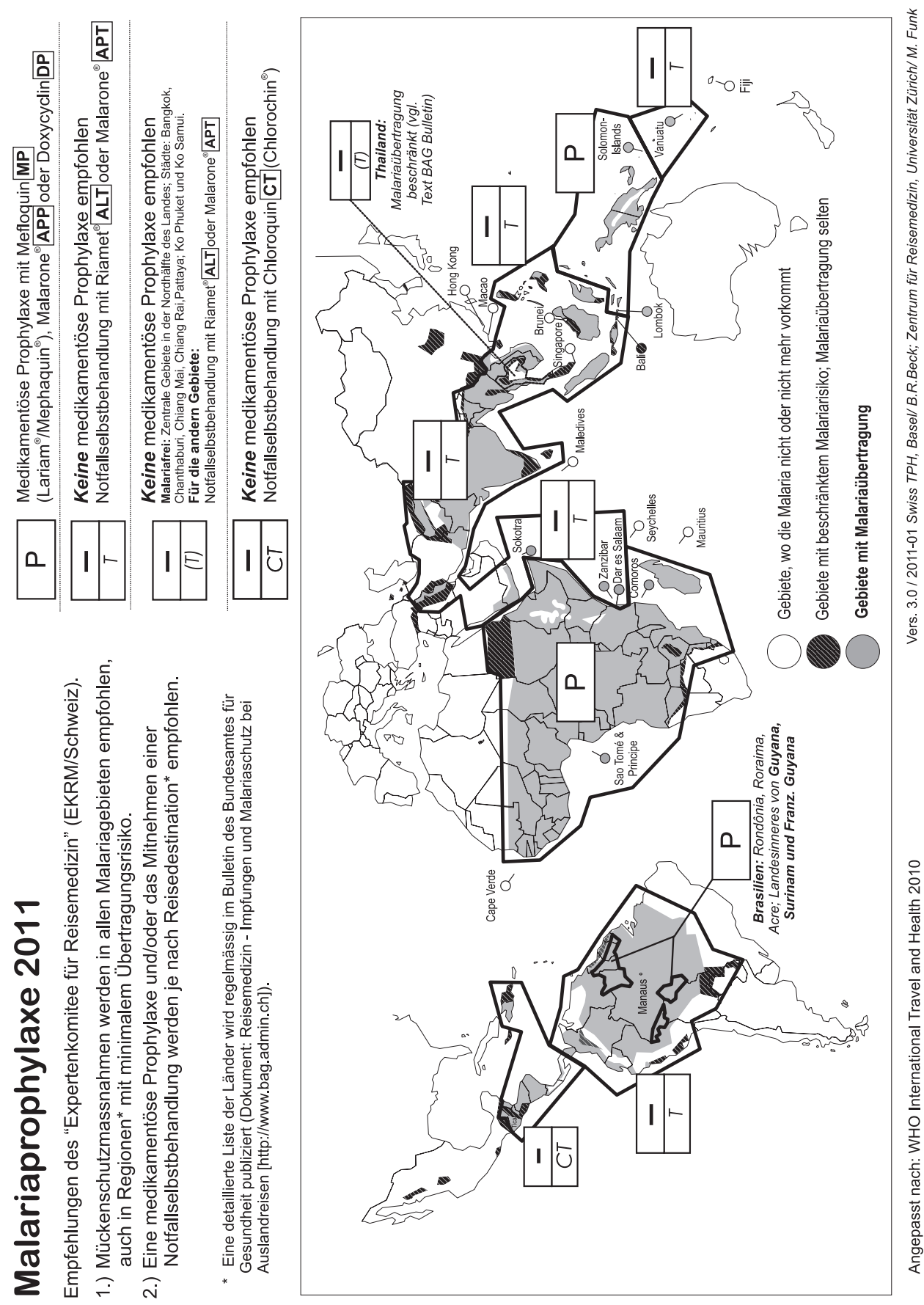

Abb. 8.5: Malariagebiete und die für 2011 empfohlene Prophylaxemöglichkeiten des Expertenkomitees für Reisemedizin (EKRM/Schweiz) (Quelle: Beck, Tropenmedizin und Reisemedizin am Bellevue, Zürich). 
parum-Infektion ist v.a. in den ersten vier Wochen nach der Rückkehr hoch. Bei einer Vivax-Malaria ist der Symptombeginn oft später (s.a. Kap. 8.3.6).

\section{HIV/AIDS und Tuberkulose}

Die ersten AIDS-Fälle wurden 1981 bei homosexuellen Männern in den USA beschrieben. Erreger ist das Humane Immundefizienz-Virus (human immunodeficiency virus, HIV). Die Tuberkulose (Erreger: v. a. Mycobacterium tuberculosis) ist dagegen eine Erkrankung, die den Menschen schon in prähistorischer Zeit befiel. Beide Erkrankungen treten heute in vielen Regionen gemeinsam auf. Die HIV-Infektion erhöht das Risiko, sich mit Tuberkulose zu infizieren. Da die Prävalenzen beider Infektionskrankheiten vor allem in den Entwicklungsländern hoch sind, ist dort auch der Anteil an Patienten, die an beiden Krankheiten leiden, sehr hoch. Im Jahre 2009 waren weltweit $12 \%$ der 9,4 Mio. Menschen, die neu an Tuberkulose erkrankten, auch mit HIV infiziert. Achtzig Prozent dieser co-infizierten PatientInnen lebten in Afrika. Im selben Jahr war die Tuberkulose Ursache für $26 \%$ der HIV-assoziierten Todesfälle. Auf unserer LehrbuchHomepage finden Sie eine WHO-Karte, die die HIV-Prävalenz bei neuen TuberkuloseFällen im Jahr 2009 zeigt (s. Web-Abb. 8.3.1).

Anders als in Subsahara-Afrika, wo diese Krankheiten zu generalisierten Epidemien führten, betreffen sie in Europa und Nordamerika v. a. bestimmte Risikopopulationen. Die Tuberkulose wird insbesondere bei ImmigrantInnen aus Afrika und Osteuropa bzw. bei immunsupprimierten PatientInnen diagnostiziert. In der Schweiz ist die Zahl an neuen HIV-Infektionen infolge heterosexueller Kontakte oder intravenösem Drogenabusus seit Ende der 1990er Jahre stabil. Ab 2004 beobachtete man hier jedoch einen leichten Anstieg bei den Ansteckungen durch homosexuellen Kontakt, der sich inzwischen allerdings wieder zu stabilisieren scheint (Abb. 8.6). In Deutschland stieg die Zahl an Neuinfektionen Anfang des letzten Jahrzehnts nach Jahren des Rückgangs wieder deutlich an. Seit 2007 hat sich der Anstieg jedoch sichtbar verlangsamt. Insgesamt wurden im Jahr 2010 in Deutschland 2.918 neue HIV-Infektionen diagnostiziert.

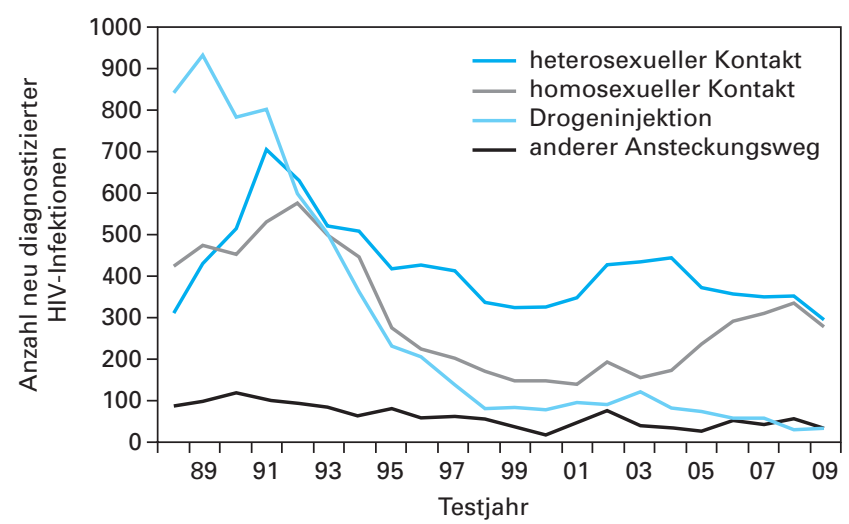

Abb. 8.6: Anzahl der neu diagnostizierten HIV-Infektionen in der Schweiz in den Jahren 1989 bis 2009, unterschieden nach verschiedenen Ansteckungswegen. Es handelt sich hierbei um statistische Schätzungen auf der Grundlage von Labor- und Arztmeldungen. (Quelle: Bundesamt für Gesundheit [BAG], Schweiz) 
Die seit 1996 zur Verfügung stehende kombinierte antiretrovirale Therapie (ART; s.a. Kap. 8.4.2) hat die HIV-assoziierte Mortalität in den industrialisierten Ländern erheblich reduziert. In Subsahara- Afrika, wo zwei Drittel der HIV-infizierten Menschen leben, wurde die ART nur zögernd ab 2001 eingeführt. Knapp zehn Jahre später bekommen immer noch weniger als 50\% der Patienten, die dringend eine Therapie brauchen, diese auch tatsächlich. Infolge der HIV-Pandemie ist die Lebenserwartung in vielen Ländern des südlichen Afrikas stark gesunken. Die katastrophalen finanziellen und sozialen Konsequenzen dieser Pandemie sind für weite Teile Afrikas noch nicht absehbar. Ohne Zweifel werden HIV und Tuberkulose in den nächsten Jahrzehnten weiterhin im Vordergrund der internationalen Public-Health-Bemühungen bleiben.

\subsubsection{Neue Infektionskrankheiten}

Im Jahr 1969 verkündete William Stuart, der Leiter der United States Public Health Services: „Es ist Zeit, die Bücher über Infektionskrankheiten zu schließen." Diese Aussage spiegelt die großen Errungenschaften bei der Prävention und Behandlung von Infektionskrankheiten in der ersten Hälfte des 20. Jh. wider, wie z.B. die Einführung von verbesserten Hygienemaßnahmen (sanitäre Einrichtungen, Verbesserung der Wasserqualität etc.), die Entdeckung von Antibiotika und die Einführung von Impfungen. Im Nachhinein zeigte sich jedoch, dass diese Einschätzung zu optimistisch war. Seither sind zahlreiche neue Infektionskrankheiten wie etwa HIV/AIDS (s. Kap. 8.3.2) aufgetreten, es kam zu einer erneuten Zunahme von bekannten Infektionen wie etwa der Tuberkulose oder des Denguefiebers. Auch die steigende Zahl der Antibiotikaresistenzen (s. Kap. 8.3.6) hat deutlich gezeigt, dass sich Infektionserreger dank der ständigen Veränderung ihrer genetischen Merkmale (Evolution) unseren Kontrollmaßnahmen entziehen können.

Der Begriff der neuen Infektionskrankheiten (Emerging Infections) umfasst neue, bisher unbekannte Infektionen, neue Varianten einer bekannten Infektion sowie wieder neu auftretende Infektionskrankheiten mit hohem epidemischem oder endemischem Potential. Abb. 8.7 zeigt die wichtigsten Faktoren, die zu einer Zu- bzw. Abnahme von Infektionskrankheiten führen.

Die meisten neuen oder wieder neu auftretenden Infektionskrankheiten sind vektorübertragene Erkrankungen, wie die Lyme-Borreliose, die Malaria, das Dengue-Fieber und Chikungunya, sowie Zoonosen wie SARS, Vogelgrippe und Ebola (s. Kap. 8.3.4). Die Entstehung solcher Infektionskrankheiten wird vor allem durch die sozioökonomischen und ökologischen Faktoren beeinflusst, die an einem Ort herrschen. Die zunehmende Migration, die Ausbreitung städtischer Lebensformen und andere Veränderungen in der Umwelt der Menschen ermöglichten es Bakterien und Viren, sich noch besser an den Menschen anzupassen. Sie erhöhen entweder die Empfindlichkeit des Menschen für bestimmte Krankheitskeime, oder sie steigern die Wahrscheinlichkeit der Exposition gegenüber einem Mikroorganismus bzw. dessen Übertragung (Transmission). Ein Beispiel dafür ist die Zunahme von bakteriellen Infektionskrankheiten in den industrialisierten Ländern. Hier führten die Verbesserungen der medizinischen Technik zu einem Anstieg der Lebenserwartung in der Bevölkerung allgemein, insbesondere jedoch zu einer erhöhten Überlebensrate von chronisch kranken und immunsupprimierten Patienten. Dadurch erhöhte sich auch die Zahl der für bakterielle Infekte empfindlichen Personen. 

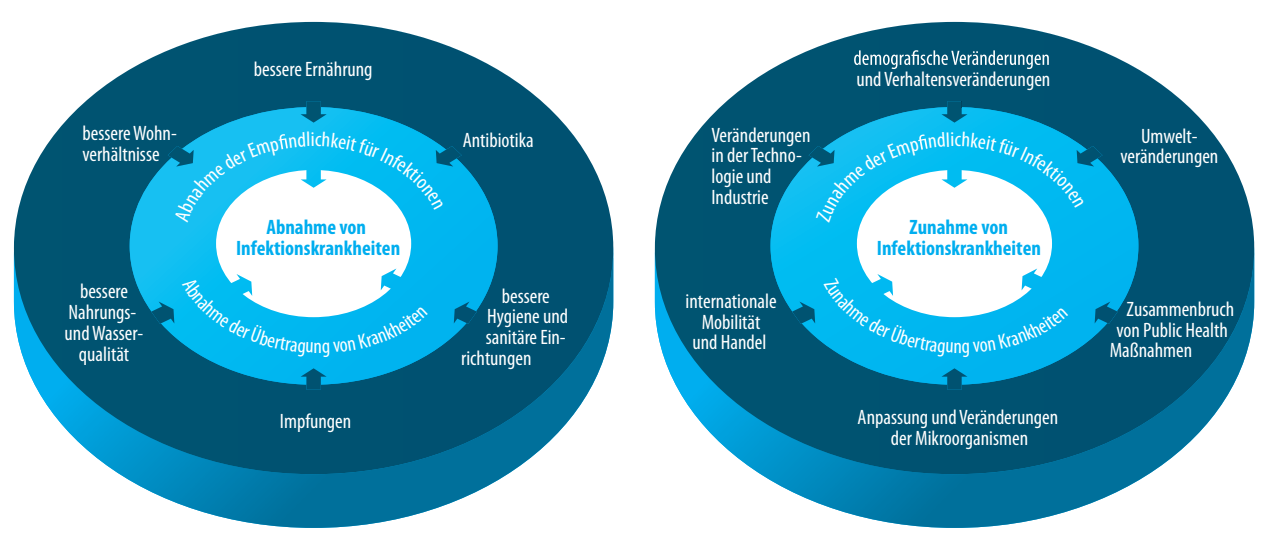

Ablb. 8.7: Faktoren, die zu einer Ab- bzw. Zunahme von Infektionskrankheiten führen. Die Bedingungen, die zu einer Zunahme von Infektionskrankheiten führen, begünstigen das Auftreten von neuen Infektionskrankheiten (Emerging Infections). (Quelle: modifiziert nach Cohen ML. Changing patterns of infectious disease. Nature 2000; 406: 762-767)

Die durch die Globalisierung bedingte Zunahme der Reisetätigkeit und des internationalen Austauschs hat sowohl die Geschwindigkeit als auch das Ausmaß der Verbreitung von Mikroorganismen massiv gefördert (s. Kap. 8.3.7). Dies hat zu einem Anstieg der Zahl an Krankheitsausbrüchen bei verschiedenen Infektionserkrankungen geführt. Eine globale, gemeinsame Public-Health-Antwort auf dieses Phänomen ist zwingend nötig, um die Anzahl und Dauer dieser Epidemien sowie deren Konsequenzen kontrollieren zu können. Die beiden wichtigsten Beispiele neuer Infektionskrankheiten sind HIV/AIDS (s. Kap. 8.3.2) und SARS.

\section{SARS}

Am 13.03.2003 teilte die WHO ihren Verdacht mit, dass sich eine neue, hochgradig ansteckende Infektionserkrankung ausbreitete, die man später als SARS (Severe Acute Respiratory Syndrome, Schweres Akutes Atemwegssyndrom) bezeichnete. SARS nahm als Infektion beim Menschen seinen Ursprung in der chinesischen Provinz Guandong. Seine weltweite Verbreitung startete mit der Reise eines bereits erkrankten Arztes nach Hongkong. Bei dessen Aufenthalt in einem Hotel infizierte er dort mindestens 10 Personen, die die Infektion anschließend nach Vietnam, Singapur, in die USA, nach Kanada und Irland exportierten. Der Arzt (Indexfall) war demnach ein sog. Superspreader („Superverbreiter", s. Tab. 8.3), da die durchschnittliche basale Reproduktionsrate $R_{0}$ später nur auf 3 geschätzt wurde. Zwischen November 2002 und Juni 2003 kam es weltweit zu insgesamt 8.456 SARS-Fällen mit einer geschätzten Letalität von 9-10\%. Als Erreger wurde innerhalb weniger Monate ein neuartiges Coronavirus identifiziert, dessen Reservoir wahrscheinlich Wildkatzen sind. Dank rascher, rigoroser internationaler Kontrollmaßnahmen, die vorwiegend eine Expositionsprophylaxe beinhalteten (s. Kap. 8.4.3), konnte die Weiterverbreitung von SARS gestoppt werden. 


\subsubsection{Zoonosen und vektorübertragene Infektionskrankheiten}

Zoonosen sind Infektionen, die auf natürliche Weise zwischen Wirbeltieren und Menschen übertragen werden. Vektorübertragene Infektionskrankheiten sind Krankheiten, die durch einen lebenden Vektor (Träger; häufig: Insekten) auf den Menschen übertragen werden. Bei einigen Infektionen ist sowohl ein Wirbeltier als auch ein Vektor am Übertragungszyklus (Transmissionszyklus) beteiligt. In diesem Fall sind sowohl Mensch als auch Tier Hauptwirte ${ }^{26}$ (z.B. bei der Schlafkrankheit), oder der Mensch ist - wie etwa bei der Pest - nur als akzidenteller Wirt (Fehlwirt, Gelegenheitswirt) betroffen.

Einige der Zoonosen und vektorübertragenen Infektionskrankheiten, wie die Malaria (s. Kap. 8.3.2) und das Dengue-Fieber, gehören zu den häufigsten Infektionserkrankungen weltweit. Das Interesse an Zoonosen und vektorübertragenen Infektionen hat seit dem Ende des 20. Jh. wieder zugenommen, da der überwiegende Anteil von neu entdeckten oder erneut auftretenden Infektionen (Emerging Infections) ihren Ursprung in der Tierwelt haben. Viele dieser Infektionskrankheiten gehören auch zu den so genannten Neglected Tropical Diseases (NTDs). Dies sind Krankheiten, die vor allem in nicht industrialisierten Ländern endemisch sind und dort in den armen Bevölkerungsgruppen eine hohe Krankheitslast verursachen. Auf unserer Lehrbuch-Homepage zeigt WebAbb. 8.3.2 die geographische Verteilung der wichtigsten NTDs. Da sie in den industrialisierten Ländern praktisch nicht vorkommen, wurden diese Krankheiten lange Zeit von der internationalen Gemeinschaft vernachlässigt. Heute unterstützen die WHO und andere Organisationen Initiativen gegen NTDs. Die globale Kontrolle der NTDs ist ein wichtiger Beitrag zur Bekämpfung der Armut und ein notwendiger Schritt zur Erreichung der "Millenium-Entwicklungsziele" (s. Kap. 9.3.1).

Zahlreiche virale, bakterielle und parasitäre Infektionskrankheiten sind Zoonosen oder vektorübertragene Infektionskrankheiten. Zoonosen sind geographisch recht unterschiedlich verteilt. Auch in gemäßigten Klimazonen und in industrialisierten Ländern spielen sie eine bedeutende Rolle. Auf unserer Lehrbuch-Homepage finden Sie in WebTab. 8.3.1 die Erreger und Wirte von Tollwut, Toxoplasmose, Milzbrand und anderen weltweit wichtigen Zoonosen. Anders als Zoonosen kommen vektorübertragene Parasiten hingegen häufiger in tropischen Gebieten und in nicht industrialisierten Ländern vor. Zu den wichtigsten vektorübertragenen Infektionskrankheiten gehören die Malaria, die Schlafkrankheit, die Leishmaniose, das Dengue-Fieber und die Borreliose (s. WebTab. 8.3.2 auf unserer Lehrbuch-Homepage).

\section{Durch Zecken übertragene Krankheiten}

Während in tropischen Ländern Stechmücken die häufigsten Vektoren sind, überwiegen in Europa und Nordamerika Zecken als Überträger. Zecken sind u. a. für die Transmission von verschiedenen Arboviren, Borrelien und Rickettsien verantwortlich. Die Arthropoden halten sich überwiegend in hohen Gräsern, im Gestrüpp oder im Unterholz auf. Wenn ein potentieller Wirt sie streift, heften sie sich an seine Haut und übertragen beim Blutsaugen die Krankheitserreger auf Mensch oder Tier. In Europa werden vor

${ }^{26}$ Hauptwirt (= Endwirt): Lebewesen, das von Parasiten aufgrund der optimalen Entwicklungsund Vermehrungsbedingungen bevorzugt befallen wird. Zwischenwirt (= Intermediärwirt): Organismus, in dem der Parasit seine Entwicklung fortsetzt, ohne geschlechtsreif zu werden 
allem die Erreger der Lyme-Borreliose und der Frühsommer-Meningoenzephalitis (FSME) durch Zeckenstiche übertragen.

Die Lyme-Borreliose (Lyme disease) ist eine durch Borrelien verursachte systemische Infektionskrankheit. In der Regel ist eine antibiotische Behandlung dann erfolgreich, wenn die Diagnose frühzeitig gestellt wird. Das sog. Post-Lyme-Syndrom, das mit chronischer Müdigkeit sowie Muskel- und Gelenkschmerzen einher geht und trotz adäquater Therapie der Borreliose auftreten kann, ist in seiner Entstehung im Detail bislang noch nicht geklärt. Da vereinzelt Borelliose-Patienten trotz adäquater Therapie über persistierende Beschwerden klagen, wurde die Lyme-Borreliose in den letzten Jahren zu einer populären Erklärung für unklare, chronische Beschwerden.

Das FSME-Virus, ein Flavivirus, kann beim Menschen zu einem grippalen Syndrom und seltener auch zu einer schweren Infektion des Zentralnervensystems führen. Im Gegensatz zur Lyme-Borreliose, die in Deutschland, Österreich und der Schweiz ubiquitär vorkommt, sind bei der Frühsommer-Meningoenzephalitis spezifische Endemiegebiete bekannt (s. Abb. 8.8). In den letzten 30 Jahren wurde eine kontinuierliche Zunahme der Fallzahlen registriert. Als mögliche Ursache dieses Anstiegs kommt die globale Erwärmung in Frage (s. Kap. 5.5), da sich die Zecken bei höheren Durchschnittstemperaturen besser entwickeln und dadurch auch weiter verbreiten können.

\section{Kontrollmaßnahmen bei Zoonosen und vektorübertragenen Infektionskrankheiten}

Die gezielte Kontrolle von Zoonosen und vektorübertragenen Infektionskrankheiten verlangt detaillierte Kenntnisse über die komplexen Interaktionen zwischen Menschen, Wirbeltieren, Insekten und Mikroorganismen. Zur Bekämpfung von Zoonosen und vektorübertragenen Infektionen bieten sich neben den Maßnahmen am Menschen auch Maßnahmen zur Kontrolle des Tierreservoirs bzw. Vektor-Kontrollmaßnahmen an.

Beispiele für solche Programme sind

- die Verbreitung von Insektizid-imprägnierten Moskitonetzen gegen Malaria in Afrika (s. Kap. 8.3.2).

- die Maßnahmen zur Verminderung der Aedes-Mückenlarvengewässer und das Einsetzen von Larviziden sowie larvenfressenden Fischen zur Bekämpfung des Denguefiebers in Zentral- und Südamerika

- die Anpassung der Umgebung, z. B. das Roden von Waldrändern in Ostafrika, um eine Distanz zwischen den Wohngebieten und den im Wald lebenden Tsetsefliegen zu schaffen, die die Trypanosomen (Erreger der Schlafkrankheit) übertragen

- die Programme zur Bekämpfung von Salmonelleninfektionen bei Hühnern in Europa

Die Existenz mehrerer biologischer Reservoire ist ein großes Hindernis für die Ausrottung (Eradikation) von Zoonosen und vektorübertragenen Infektionskrankheiten. Die Durchführung von Kontrollmaßnahmen verlangt hier eine enge Zusammenarbeit zwischen den verschiedenen Experten für Umweltwissenschaften sowie für Infektionskrankheiten bei Mensch und Tier. Das Bestreben, eine solche umfassende Zusammenarbeit der Disziplinen herbeizuführen, wird gemäß dem Motto der Initiative "One World-One Medicine-One Health" unter dem Begriff "One health" zusammengefasst. 


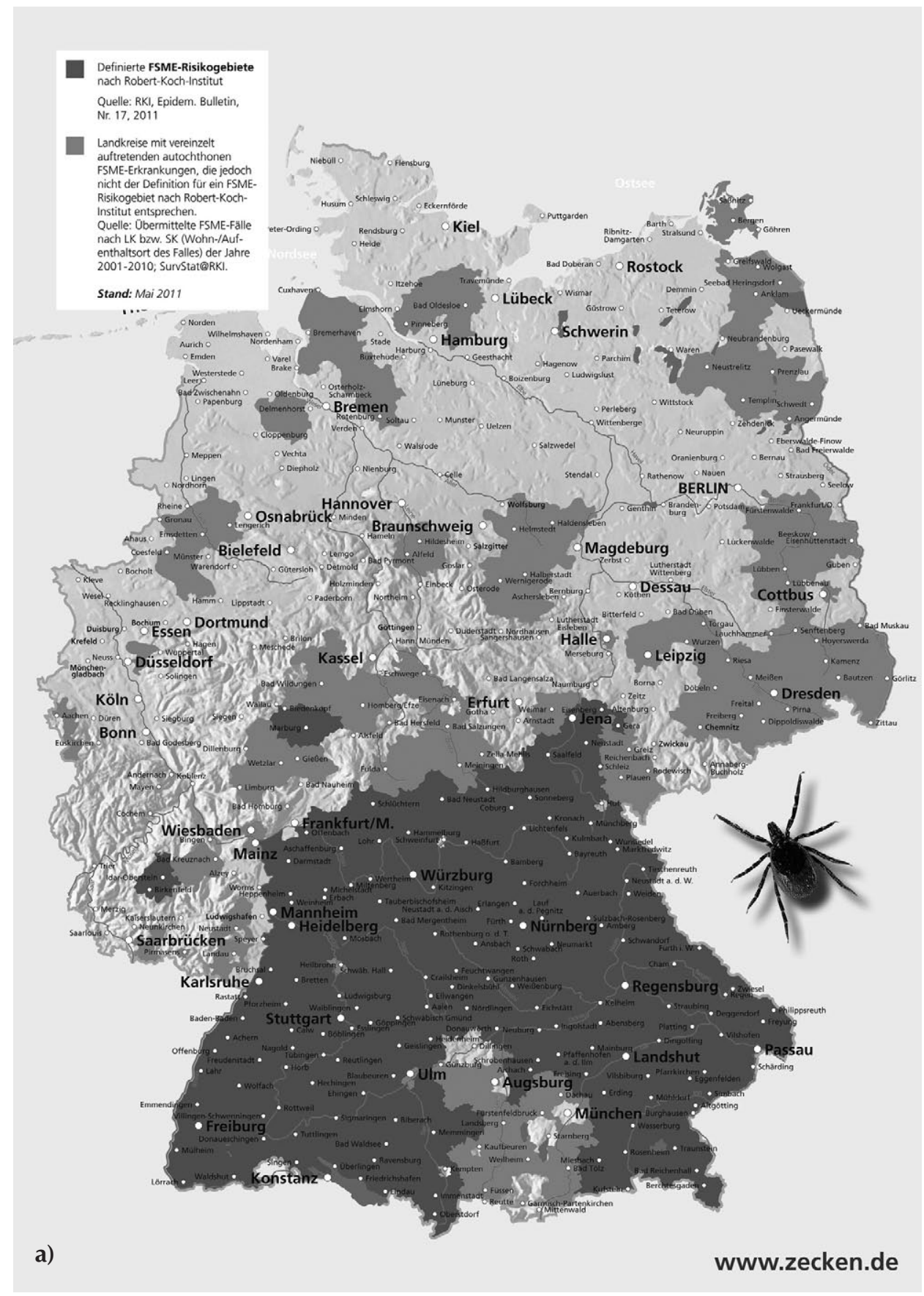

Abb. 8.8: FSME-Risikogebiete in Deutschland (a) (Quellen: www.zecken.de). 


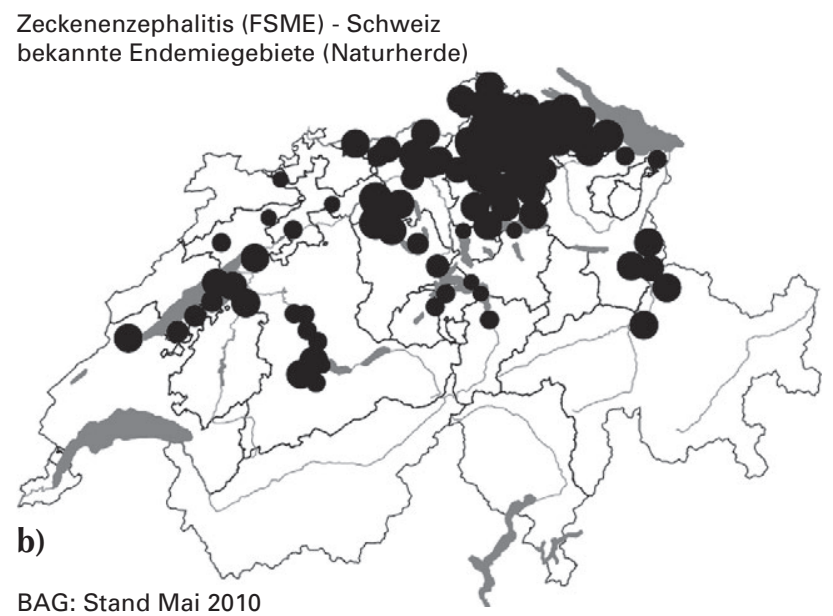

Abb. 8.8: FSME-Risikogebiete in der Schweiz (b) (Quellen: Bundesamt für Gesundheit BAG www. bag.admin.ch/infekt).

\subsubsection{Nosokomiale Infektionen}

Als nosokomiale Infektion (aus dem Altgriechischen von nósos: Krankheit und komein: pflegen; auf Deutsch: Krankenhausinfektion) bezeichnet man eine Infektion, die in einer Gesundheitseinrichtung erworben wurde. Der Begriff Gesundheitseinrichtung umfasst dabei neben den Akutkrankenhäusern auch die Einrichtungen der Langzeitpflege sowie ambulante Praxen.

\section{Definition}

Überwachungsprogramme definieren nosokomiale Infektionen als Infektionen, die bei Aufnahme in eine Gesundheitseinrichtung noch nicht latent vorhanden waren, nach mehr als 48 Stunden dann aber manifest (bzw. diagnostiziert) wurden. Das Intervall von 48 Stunden ergibt sich daraus, dass nosokomiale Infektionen häufig durch Bakterien ausgelöst werden, und die Inkubationszeit von bakteriellen Infektionen meist weniger als 48 Stunden beträgt. Bei Infektionskrankheiten mit einer längeren Inkubationszeit (z. B. Legionellose oder Erkrankungen, die durch respiratorische Viren hervorgerufen werden) muss diese Definition dementsprechend angepasst werden. Nosokomiale Infektionen können auch erst nach einer Krankenhausentlassung manifest werden - ein Aspekt, der für Überwachungsprogramme besonders relevant ist (s.u.). Für die einzelnen nosokomialen Infektionen haben die Centers for Disease Control and Prevention (CDC) in den USA spezifische Definitionen entwickelt, die inzwischen weltweit gebräuchlich sind.

\section{Bedeutung}

Nosokomiale Infektionen sind von großer gesundheitspolitischer Bedeutung. Sie gefährden nicht nur die Behandlungsqualität und die Patientensicherheit (s. Kap. 3.4), sondern verursachen zusätzlich auch erhebliche Kosten. Die Kosten entstehen durch 
einen verlängerten Aufenthalt im Krankenhaus - im Durchschnitt resultiert daraus eine Verdoppelung der Krankenhaustage - und durch zusätzliche Behandlungen. Weitere Kosten fallen darüber hinaus durch den Arbeitsausfall sowie durch Betreuungsleistungen von Angehörigen oder Pflegekräften an. Besonders hoch sind die Folgekosten bei Invalidität oder Tod. Eine Schätzung der durchschnittlichen Mehrkosten ist jedoch schwierig, da diese je nach Art und Schweregrad der Infektion sowie der Art der Vergütungsregelung stark schwanken können.

\section{Häufigkeit}

In den Schweizer Akutkrankenhäusern wird die Prävalenz nosokomialer Infektionen auf durchschnittlich 7\% der infizierten Patienten geschätzt. Ihre Anzahl ist mit 4-5\% in den kleinen Krankenhäusern $(<200$ Betten) wesentlich niedriger als in den Großkliniken (> 500 Betten), wo sie 10-11\% beträgt. Innerhalb eines Krankenhauses beobachtet man die höchste Rate an nosokomialen Infektionen auf den Intensivstationen. Hier gehen etwa $25 \%$ der Infektionen auf Krankenhauskeime zurück (s. www.swiss-noso.ch).

In Deutschland wurden für das Jahr 2006 Schätzungen zur Häufigkeit von nosokomialen Infektionen und zu Todesfällen infolge nosokomialer Infektionen vorgenommen. Danach kam es in diesem Jahr zu 400.000 bis 600.000 nosokomialen Infektionen. Bei geschätzten 10.000 bis 15.000 PatientInnen waren nosokomiale Infektionen die Todesursache. Etwa 80.000 bis 180.000 dieser nosokomialen Infektionen und 1.500 bis 4.500 der Todesfälle infolge nosokomialer Infektionen sind pro Jahr nach einer hierauf basierenden Hochrechnung vermeidbar.

\section{Klinische Manifestation}

Die häufigste Form von Krankenhausinfektionen sind chirurgische Wundinfektionen (ca. 30\%) und symptomatische Harnwegsinfekte (ca. 20\%), gefolgt von Sepsis/Bakteriämie $(15 \%)$ und Pneumonie (13\%). Manche Überwachungsprogramme schließen auch asymptomatische Harnwegsinfektionen mit ein, wodurch Harnwegsinfekte in der Statistik auf den ersten Rang rücken.

\section{Infektionserreger}

Bei Erwachsenen werden Krankenhausinfektionen mit Abstand am häufigsten durch Bakterien verursacht. In der Pädiatrie sind darüber hinaus auch nosokomiale Virusinfektionen von Bedeutung. Sie zeigen allerdings saisonale Schwankungen parallel zur epidemiologischen Situation in der Gesamtbevölkerung. Die häufigsten bakteriellen Erreger von Krankenhausinfektionen sind Staphylococcus aureus (10-15\%), koagulasenegative Staphylokokken (10-15\%) und Escherichia coli (10-15\%). Eine wichtige Rolle spielen auch antibiotikaresistente Erreger wie methicillinresistente S. aureus (MRSA) oder Enterobacteriaceae, die zur Bildung von Breitspektrum-Betalaktamasen in der Lage sind (ESBL; s. Kap. 8.3.6). Zu den häufigsten nosokomial übertragenen Viren gehören neben Respiratory Syncytial Virus (RSV) und Influenzavirus auch Rota- und Noroviren.

\section{Quelle}

Die weitaus häufigste Quelle für eine Krankenhausinfektion ist die patienteneigene bakterielle Flora. Die Erreger gelangen meist im Rahmen von invasiven medizinischen 
Maßnahmen (z. B. durch Beatmungsmaßnahmen oder über Venen- bzw. Harnwegskatheter) in den Körper des Patienten. Andere Quellen wie kontaminierte Gegenstände und Flächen, Wasser, Lebensmittel und Luft spielen heute in industrialisierten Ländern dank der qualitativ hoch stehenden Infrastruktur eine untergeordnete Rolle. Eine Ausnahme bilden Fälle von nosokomialen Legionellosen. Hier können die Erreger durch kontaminierte Wasserleitungen übertragen werden.

\section{Wichtige Risikofaktoren}

Es gibt eine Reihe von Faktoren, die das Risiko erhöhen, an einer nosokomialen Infektion zu erkranken. Man unterscheidet hierbei exogene, nicht patienten-gebundene von endogenen, patienten-gebundenen Risikofaktoren. Endogene Faktoren, die das Infektionsrisiko erhöhen, sind v.a. Vorerkrankungen wie etwa Stoffwechselstörungen und Krankheiten, bei denen es zu einer Immunsuppression kommt, aber auch eine vorhandene Adipositas und der Konsum von Suchtmitteln (z. B. Nikotin). Exogene Risikofaktoren entstehen in der Regel im Rahmen von therapeutischen Maßnahmen. An erster Stelle stehen hier Eingriffe, die die natürliche Infektabwehr der Patienten beeinträchtigen können (das Legen eines intravaskulären Katheters oder eines Harnwegskatheters, die künstliche Beatmung etc.).

Die Korrelation zwischen der Rate an nosokomialen Infektionen und der Größe bzw. Art des Krankenhauses spiegelt einerseits die dort angebotenen Behandlungen, andererseits die unterschiedliche Zusammensetzung der Patientenpopulation im Hinblick auf die Prävalenz von Risikofaktoren wider („Patientenmix“). Patienten in größeren Kliniken weisen in der Regel eine höhere Komorbidität auf. Darüber hinaus werden in größeren Krankenhäusern bzw. Kliniken der Maximalversorgung komplexere und invasivere Behandlungen durchgeführt. Diese Unterschiede müssen bei Vergleichen zwischen den Inzidenzraten verschiedener Krankenhäuser oder den Inzidenzraten, die in einer Klinik zu verschiedenen Zeiten erhoben wurden, berücksichtigt werden.

\section{Kontrollstrategien}

Programme zur Kontrolle von Krankenhausinfektionen beruhen auf mehreren Pfeilern (s. Tab. 8.4). Auf unserer Lehrbuch-Homepage finden Sie in Web-Abb. 8.3.3 Näheres zur Überwachung chirurgischer Wundinfektionen im Rahmen des OP-KISS Krankenhaus-Infektions-Surveillance-System (www.nrz-hygiene.de).

\section{Organisationen zur Überwachung nosokomialer Infektionen}

- In Deutschland ist das Robert Koch-Institut (www.rki.de) mit dem Nationalen Referenzzentrum für die Surveillance nosokomialer Infektionen (www.nrz-hygiene.de/ surveillance/kiss/) für die Formulierung von Richtlinien zur Verhütung von Krankenhausinfektionen und die Durchführung von Überwachungsprogrammen verantwortlich.

- In der Schweiz ist dies der Verein SwissNOSO (www.swiss-noso.ch),

- in den USA die Centers for Disease Control and Prevention (CDC, www.cdc.gov) mit ihrem National Healthcare Safety Network System (NHSN). 
Tab. 8.4: Die wichtigen Pfeiler zur Kontrolle von Krankenhausinfektionen.

Hygienemaßnahmen Dies umfasst alle Handlungen und Maßnahmen, die das Risiko für eine nosokomiale Infektion sowie die Übertragung von Erregern einer Krankenhausinfektion vermindern. Hierzu gehören neben den Standardhygienemaßnahmen auch Hygienemaßnahmen bei Eingriffen am Patienten, eine prophylaktische Behandlungen (z. B. die perioperative Antibiotikaprophylaxe) und die Behandlung von Instrumenten, Gegenständen und Flächen. Im weiteren Sinne gehören dazu auch personalärztliche Betreuungsmaßnahmen, eine rationale Antibiotikapolitik, das Abklären von Epidemien sowie die Überwachung der Qualität von Lebensmitteln, Luft und Wasser.

Die Hygienemaßnahmen müssen schriftlich in Form von Hygienerichtlinien dokumentiert sein und regelmäßig überarbeitet werden.

Schulung

Überwachung

Verbesserung
Hierzu gehört die in regelmäßigen Abständen stattfindende Information aller relevanten Berufsgruppen im Hinblick auf die Hygienerichtlinien.

Ziel eines Überwachungsprogramms kann entweder die Ermittlung der Infektionsrate oder die Kontrolle von bestimmten Prozessen (z. B. Händehygienecompliance) sein. Infektionsraten können entweder durch Prävalenzstudien (z. B. Stichprobenerhebung in der ganzen Klinik in Bezug auf alle Infektarten) oder durch longitudinal-prospektive Studien (etwa im Hinblick auf chirurgische Wundinfektionen oder katheter-assoziierte Bakteriämien) ermittelt werden.

Erkannte Mängel (z.B. eine zu hohe Infektrate) sollen im Rahmen von Verbesserungsprojekten soweit möglich korrigiert werden. Der Erfolg dieser Maßnahmen sowie seine Nachhaltigkeit müssen überwacht werden.

\subsubsection{Antibiotikaresistenz}

\section{Definition}

Als Antibiotikaresistenz bezeichnet man die verminderte Empfindlichkeit eines Mikroorganismus gegenüber einem Antibiotikum ${ }^{27}$. Multiresistente Mikroorganismen sind Keime, die Resistenzen gegenüber mehreren wichtigen Antibiotika oder Antibiotikagruppen aufweisen. Hier sind die Therapiemöglichkeiten signifikant eingeschränkt. Der Begriff der "Krankenhausflora" umfasst Infektionserreger, die multiresistent sind und/ oder opportunistische Eigenschaften haben, d. h. nur bei einem geschwächten Wirt zu einer Erkrankung führen.

${ }^{27} \mathrm{Im}$ ursprünglichen Sinne sind Antibiotika natürlich produzierte Stoffwechselprodukte von Bakterien und Pilzen, die andere Mikroorganismen in ihrem Wachstum hemmen oder diese abtöten. Der Begriff wird heute breiter gefasst und schließt auch synthetisch hergestellt Hemmstoffe ein. 


\section{Bedeutung}

Antibiotikaresistenzen sind weltweit ein großes gesundheitspolitisches Problem, dessen Bedeutung immer mehr zunimmt. Dies haben inzwischen alle großen Organisationen mit länderübergreifenden gesundheitspolitischen Aufgaben (wie WHO, CDC und ECDC) erkannt. Sie zählen die Antibiotikaresistenz heute zu den weltweiten Problemen mit höchster Priorität.

Kosten können aufgrund einer Antibiotikaresistenz durch das Therapieversagen selbst entstehen, aber auch infolge verlängerter Krankheits- bzw. Hospitalisationsdauer, zusätzlicher Behandlungen sowie durch mögliche Invalidität oder den Tod der Betroffenen. Für die Gesundheitsinstitutionen fallen zusätzliche Kosten durch die Kontrollmaßnahmen (z. B. Isolationsmaßnahmen, Screening etc.) an.

\section{Häufigkeit/Vorkommen}

Praktisch alle wichtigen, für den Menschen pathogenen Bakterien sind heute von Antibiotikaresistenzen betroffen, es gibt jedoch auch zunehmend Resistenzen bei Viren und Parasiten. So kennt man Resistenzen gegenüber Virostatika z. B. bei HIV, Influenzaviren und dem Herpes-simplex-Virus, aber auch gegenüber antiparasitären Substanzen wie etwa bei Malariaerregern. Web-Tab. 8.3.3 auf unserer Lehrbuch-Homepage nennt Beispiele von Krankheitserregern, bei denen Resistenzen gegenüber bestimmten Antibiotika bekannt sind.

Die Verbreitung von resistenten Keimen zeigt oft geographische Unterschiede (Abb. 8.9). Methicillinresistente S. aureus-Stämme (MRSA) waren bei ihrer ersten Beschreibung im Jahr 1961 ein typisches nosokomiales Problem ohne größere Verbreitung in der Allgemeinbevölkerung. Dies galt bis in die frühen 1990er Jahre. Durch die rasche Verbreitung von ambulant erworbenen (community-acquired, CA) MRSA kam es in den letzten 10 bis 15 Jahren zu einer zunehmenden Verdrängung der nosokomialen MRSA. MRSA werden nun zunehmend auch bei Tieren beobachtet. In den Niederlanden hat sich beispielsweise zu Beginn des 21. Jahrhunderts ein spezifischer MRSA-Klon in Schweineherden ausgebreitet, der auf den Menschen übertragbar ist und inzwischen auch in anderen europäischen Ländern bei Zuchttieren beobachtet wurde.

Darüber hinaus ist die zunehmende Verbreitung von Enterobacteriaceae mit Bildung von Breitspektrum-Betalaktamasen (ESBL) und/oder Carbapenemasen (vor allem E. coli und Klebsiella pneumoniae und oxytoca) in den letzten Jahren weltweit zu einem Problem geworden. Vieles deutet darauf hin, dass die zunehmende Resistenz in dieser Bakterienfamilie von Umweltkeimen auf humanpathogene Keime übertragen wird und sich die resistenten Keime in Gesundheitsinstitutionen ebenso wie im ambulanten Bereich ausbreiten. Als Reservoire dienen neben dem Menschen wahrscheinlich auch Tiere und Lebensmittel.

\section{Risikofaktoren}

Der wichtigste Risikofaktor, der die Selektion von antibiotikaresistenten Keimen begünstigt, ist die unkritische Anwendung von Antibiotika. Eine bedeutende Rolle spielt hier neben dem Antibiotikaeinsatz beim Menschen auch die Verwendung von Antibiotika in der Tierzucht. In Europa ist die Gabe von Antibiotika zur Wachstumsförderung bei Tieren seit Beginn des 21. Jahrhunderts verboten. Antibiotika werden hier jedoch 


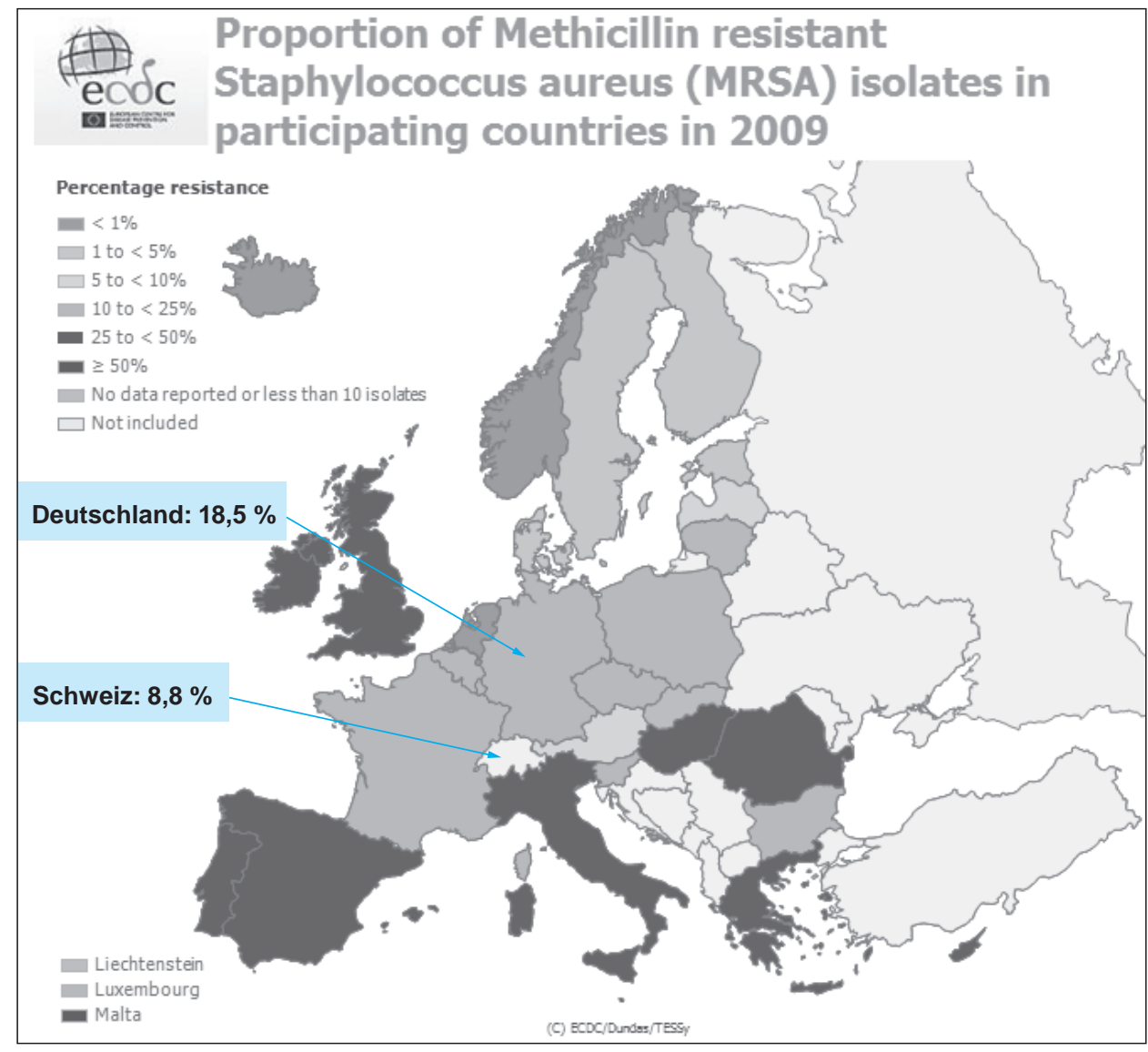

Abb. 8.9: Prävalenz der Methicillinresistenz bei Staphylococcus aureus in Europa im Jahr 2008. Die Überwachung der Antibiotikaresistenzen erfolgt in weiten Teilen Europas über das EARS Programm (www.ecdc.europa.eu) und in der Schweiz über das nationale Programm ANRESIS (www.anresis.ch). Die Werte für Deutschland und die Schweiz wurden ergänzt (Quelle modifiziert nach: European Centre for Disease Prevention and Control. Antimicrobial resistance surveillance in Europe 2009. Resistance Surveillance Network (EARS-Net). Stockholm: ECDC; 2010).

weiterhin zu therapeutischen Zwecken eingesetzt. Es kommen dabei auch Substanzen und Substanzklassen zur Anwendung, die in der Humanmedizin verwendet werden.

Ein weiterer wichtiger Faktor, der bei der Entstehung resistenter Mikroorganismen eine Rolle spielt, ist die Übertragung von Mensch zu Mensch bzw. von anderen Reservoiren auf den Menschen und umgekehrt. Von Bedeutung ist hierbei das Verhalten der Menschen, wahrscheinlich aber auch die genetisch bedingte Empfänglichkeit (Suszeptibilität) von Wirt und Keim. 


\section{Kontrollstrategien}

Die Kontrolle von Antibiotikaresistenzen ruht auf drei Pfeilern.

- Überwachungsprogramme zur Antibiotikaresistenz erlauben, das Problem zu quantifizieren, sowie Zeittrends und Gebiete bzw. Bevölkerungsgruppen mit erhöhtem Risiko zu erkennen. Sie bilden deshalb die Basis einer zielgerichteten Kontrolle sowie für die Überprüfung des Erfolgs von Maßnahmepaketen (Kontrollprogramme). Überwachungsprogramme unterstützen zudem Therapeutlnnen bei der korrekten Wahl eines Antibiotikums, da sie ihnen Auskunft über die Resistenzepidemiologie geben.

- Antibiotikagabe: Eine besonders wichtige Maßnahme zur Eingrenzung der Resistenzproblematik ist die kontrollierte Anwendung von Antibiotika. Einer der Gründe hierfür ist, dass Antibiotika unterschiedlich breite Wirkspektren aufweisen und sich in ihrer Fähigkeit unterscheiden, Resistenzen auszubilden. Darüber hinaus führt eine zu niedrige Dosierung nicht nur zum Therapieversagen, sondern fördert auch die Selektion von resistenten Keimen. Eine zu lange Therapiedauer trägt ebenfalls zur Resistenzselektion bei, ohne dass ein weiterer Nutzen für den Therapieerfolg zu verzeichnen wäre.

- Expositionsprophylaxe (s.a. Kap. 8.4.3): Kontrollmaßnahmen zur Verhinderung der Übertragung von resistenten Keimen von Mensch zu Mensch haben sich vor allem im Krankenhausbereich als effizient erwiesen. Sie beinhalten Standardhygienemaßnahmen bei allen Patienten sowie die Kontaktisolation von Keimträgern. Falls möglich, sollte dies mit einem aktiven Screening für Träger resistenter Keime kombiniert werden. Dies hängt jedoch davon ab, ob ein solcher Screening-Test verfügbar ist.

Mit Hilfe dieses Verfahrens kann z.B. die MRSA-Prävalenz in einer Gesundheitseinrichtung signifikant gesenkt werden. Dagegen ist die Wirksamkeit von Hygienemaßnahmen zur Verminderung von Antibiotikaresistenzen im ambulanten Bereich bislang noch nicht genügend untersucht.

\subsubsection{Reisemedizin}

Reisen in tropische Gebiete und sog. Entwicklungsländer sind mit einem erheblichen zusätzlichen Krankheitsrisiko verbunden. Infektionskrankheiten haben hierbei den höchsten Stellenwert. Aber auch Unfälle, Herz- und Kreislaufprobleme, die Verschlechterung einer bestehenden Grundkrankheit sowie psychiatrische Störungen treten häufiger auf.

Verschiedene Faktoren beeinflussen das Infektionsrisiko auf Reisen:

- das Reiseziel

- ein erhöhtes Expositionsrisiko durch mangelnde Hygiene im bereisten Gebiet

- die unterschiedliche Infektionsepidemiologie beispielsweise bei Parasiten, vektorübertragenen oder sexuell übertragenen Infektionskrankheiten

- die Jahreszeit (Beispiel: erhöhtes Malaria-Risiko während der Regenzeit)

- die Reisedauer (Beispiel: erhöhtes Tollwut-Risiko bei längerem Aufenthalt in einem Risikogebiet) 
- die Unterkunft (Beispiel: infektiöse Hauterkrankungen)

- risikoreiches Verhalten (Beispiel: Sexualkontakte)

- eine erhöhte Empfindlichkeit durch Stress, klimatische Bedingungen etc.

- genetische Faktoren (Beispiel: Geschlecht)

In den letzten Jahren ist die Bedeutung der auf Reisen erworbenen Infektionen durch die zunehmende beruflich bedingte und private Reisetätigkeit der Menschen erheblich angestiegen. Man schätzt, dass jährlich über 20 Mio. Deutsche in Gebiete verreisen, die ein gesundheitliches Risiko bergen. Im Jahr 2001 besuchten 1,3 Mio. Schweizer ein tropisches Land.

Reiseinfektionen betreffen natürlich die Reisenden selbst. Je nach Art des Infektionserregers kann der Import von Infektionskrankheiten durch infizierte bzw. kolonisierte Reisende oder durch mitgeführte Produkte jedoch nach der Rückkehr ins Ursprungsland auch zu einer weiteren, eventuell sogar epidemischen Verbreitung des Erregers führen.

Die Prävention von Reiseinfektionen basiert auf drei Pfeilern:

- Expositionsprophylaxe (s. Kap. 8.4.3, Kap. 8.3.6. und Reisediarrhöe, s.u.)

- Impfung (s. Kap. 8.4.1 und Text s.u.)

- Chemoprophylaxe (s. Kap. 8.4.2, Kap. 8.3.2 und Reisediarrhöe, s.u.)

Die Vermittlung dieser präventiven Prinzipien geschieht ebenso wie die präventive Behandlung in den meisten industrialisierten Ländern im Rahmen von reisemedizinischen Sprechstunden bzw. Beratungen. Dies kann sowohl durch Spezialisten für Tropenkrankheiten oder durch entsprechend geschulte ÄrztInnen anderer Fachrichtungen an einem Zentrum für Tropenmedizin oder in der Praxis stattfinden. Einige Maßnahmen, wie etwa die Gelbfieberimpfung in der Schweiz, benötigen eine spezielle Praxisbewilligung. Auch in Deutschland darf die Gelbfieberimpfung nur durch speziell weitergebildete ÄrztInnen verabreicht werden. Da Impfungen eine gewisse Zeit benötigen, um ihre Schutzwirkung aufzubauen, und je nach Impfung mehrere Dosen notwendig sein können, muss die Beratung in einem ausreichend langen Zeitabstand vor der Abreise stattfinden. Wichtig ist darüber hinaus, dass die Reisenden über Präventivmaßnahmen und Behandlungsmöglichkeiten bei den in Frage kommenden Infektionen verständlich informiert werden, und wissen, wann sie im Notfall medizinische Hilfe in Anspruch nehmen sollen.

Zum Thema Reisemedizin bietet das Internet Reisenden und Ärzten über verschiedene Quellen ausführliches, beratendes Material an. Informationen finden sich z. B. bei Tropimed (www.tropimed.com), safetravel (www.safetravel.ch), dem Schweizerischen Bundesamt für Gesundheit (www.bag.admin.ch), dem FORUM Reisen und Medizin (www.frm-web.de), dem Robert Koch-Institut (www.rki.de), den CDC (www.cdc.gov/ travel) und der WHO (www.who.int/ith).

Im Folgenden werden beispielhaft einige wichtige Themen aus dem Bereich der Reisemedizin ausführlicher besprochen. 


\section{Impfungen}

Eine reisemedizinische Beratung sollte als wichtige Gelegenheit genutzt werden, alle empfohlenen Routineimpfungen auf ihre Aktualität hin zu überprüfen! Insbesondere sollen der Schutz vor Diphtherie, Tetanus, Poliomyelitis und Masern kontrolliert werden. Daneben gibt es eine Reihe von Indikationsimpfungen, die speziell bei Reisenden durchgeführt werden. Dazu gehören Impfungen gegen Hepatitis A (meist kombiniert mit einer Hepatitis B-Impfung), gegen Tollwut, Japanische Encephalitis, Abdominaltyphus, Gelbfieber und Infekte durch Meningokokken bestimmter Serogruppen. Einige dieser Impfungen werden von bestimmten Zielländern obligatorisch bei der Einreise verlangt. Beispiele hierfür sind die Gelbfieber-Impfung in bestimmten tropischen Regionen Afrikas und Südamerikas sowie die Meningokokken-Impfung (Serogruppen A, W 135 und Y) bei Mekka-Pilgern.

\section{Reisediarrhöe}

Das häufigste Gesundheitsproblem von Tropenreisenden bzw. Reisenden in sog. Entwicklungsländern ist der Reisedurchfall. Ungefähr die Hälfte der Reisenden leidet an Durchfallerkrankungen. Wie auch bei anderen Infektionen hängt das individuelle Risiko, eine Reisediarrhöe zu entwickeln, von zahlreichen Faktoren ab. Hierzu gehören neben dem Reiseziel (wichtigster Prädiktor), der Jahreszeit und der Dauer des Aufenthaltes auch die Ernährung, die Art der Unterkunft und der Aktivitäten sowie wahrscheinlich auch genetische Faktoren.

Die Erreger eines Reisedurchfalls werden in der Regel fäkal-oral durch kontaminiertes Trinkwasser (s. Kap. 5.1) oder durch kontaminierte Lebensmittel übertragen. Die Mehrheit (bis zu 90\%) dieser Infektionen wird durch enteropathogene Bakterien (z. B. verschiedene E. coli-Stämme, Salmonellen, Shigellen) verursacht, nur ein kleiner Teil durch Viren (v.a. Rotaviren, Enterisches Adenovirus) oder Parasiten (z. B. Entamoeba histolytica).

Die weltweit häufigsten Erreger des Reisedurchfalls sind enterotoxische Escherichia coli. Es handelt sich dabei um eine sog. sekretorische Diarrhoe von kurzer Dauer (3-5 Tage), bei der dünnflüssige Stühle das Hauptsymptom sind. Der Schweregrad der Erkrankung ist allerdings niedrig, die Erkrankung führt in der Regel nicht zum Tod. Infektionen durch invasive pathogene Keime (wie Campylobacter, Salmonellen und Shigellen) können mit Bauchkrämpfen, blutigem Stuhlgang und Fieber einhergehen. Die durch Parasiten (Entamoeba histolytica, Giardia lamblia) verursachten Durchfallerkrankungen verlaufen in der Regel weniger akut und werden oft erst nach der Rückkehr diagnostiziert. Bei 1 bis $3 \%$ der Reisenden kommt es zu chronischen Diarrhöen (> 1 Monat Dauer).

Die Prävention der Reisediarrhoe umfasst vor allem Hygienemaßnahmen einschließlich einer geeigneten Lebensmittelhygiene (Motto: „Boil it, cook it, peel it or leave it."). Die Compliance ist bei diesen Empfehlungen allerdings gering. Eine routinemäßige Antibiotikaprophylaxe wird wegen der assoziierten Nebenwirkungen, der MedikamentenInteraktionen, dem Risiko der Entwicklung von Antibiotikaresistenzen sowie der entstehenden Kosten nicht empfohlen.

Die Reisediarrhoe ist eine meist selbstlimitierende Erkrankung. Sie benötigt keine spezifische Therapie. Allerdings muss auf eine genügende Flüssigkeits- und Elektrolytzufuhr geachtet werden. Die heute oftmals routinemäßige Verschreibung von Antibiotika 
für die Reiseapotheke muss auf der Basis der Harmlosigkeit eines Reisedurchfalls und der weltweit rasch zunehmenden Antibiotikaresistenz-Prolematik sehr kritisch hinterfragt werden.

\section{Fieber bei Reiserückkehrern}

In der Regel wenden sich Reisende mit gesundheitlichen Problemen, die sie der Reise zuschreiben, nach ihrer Rückkehr wieder an die vor Reisebeginn aufgesuchte, beratende Stelle. Nach einem Aufenthalt in den Tropen sind Fieber und Durchfälle die häufigsten Konsultationsgründe.

\section{Internet-Ressourcen}

Auf unserer Lehrbuch-Homepage (www.public-health-kompakt.de) finden Sie neben zusätzlichen Abbildungen und Tabellen Links zu weiterführender Literatur sowie zu anderen themenrelevanten Internet-Ressourcen.

\subsection{Impfungen und andere präventive Maßnahmen}

\subsubsection{Impfungen}

Impfungen gehören zu den großen medizinischen Errungenschaften des 20. Jahrhunderts. Durch sie konnten Infektionskrankheiten mit hoher Krankheitslast ausgerottet (Beispiel: Pocken) oder in ihrem Vorkommen drastisch eingeschränkt werden (Beispiele: Poliomyelitis, Diphtherie, Tetanus, Masern, Pertussis, Hepatitis B etc.). Die aktive Immunisierung spielt hierbei eine ungleich größere Rolle als die passive Immunisierung. Als aktive Immunisierung bezeichnet man die Stimulation einer Immunantwort im Wirt (z. B. im Menschen) durch Verabreichung von geeigneten Antigenen des Erregers. Solche Antigene können z. B. Eiweiße aus der Oberfläche des Erregers sein. Bei der passiven Immunisierung verabreicht man dagegen fertige Antikörper oder Immuneffektorzellen. Diese Form der Immunisierung hat jedoch nur eine beschränkte Wirkungsdauer (wenige Monate) und ist in der Regel mit hohen Kosten verbunden.

Empfehlungen zu bestimmten Impfungen werden in Deutschland und in der Schweiz durch die nationalen Gesundheitsbehörden erlassen. Unterstützt werden die Behörden dabei durch Expertengremien. In der Schweiz ist dies die Eidgenössische Impfkommission (EKIF), in Deutschland die Ständige Impfkommission (STIKO). Der auf der Basis dieser Empfehlungen erstellte Impfplan wird jährlich neuen Entwicklungen angepasst und anschließend veröffentlicht (Abb. 8.10; Schweiz: www.bag.admin.ch; Deutschland: www.rki.de).

\section{Standardimpfung, Indikationsimpfung, ergänzende Impfung}

Impfungen, die für die gesamte Bevölkerung empfohlen werden, bezeichnet man als Standard- oder Routineimpfungen (Beispiele: Impfungen gegen Tetanus, Diphtherie, Poliomyelitis, Masern).

Indikationsimpfungen werden dagegen nur Personen mit einem erhöhten Expositionsrisiko oder einem erhöhten Risiko für eine schwer verlaufende Infektion empfohlen. Beispiele hierfür sind die Impfungen gegen Influenza, Frühsommer-Meningoenzephali- 


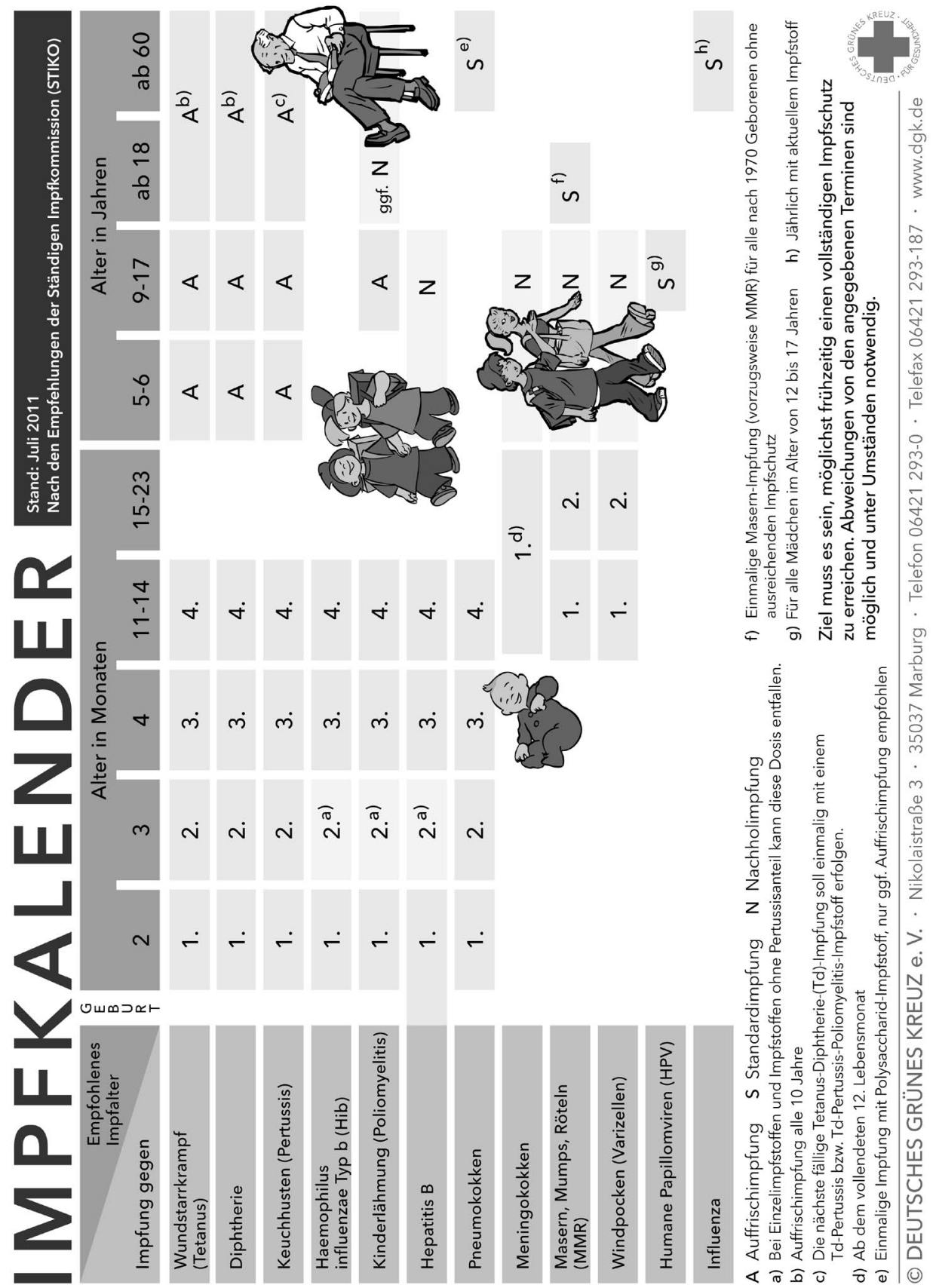

Abb. 8.10: Impfkalender nach den Empfehlungen der Ständigen Impfkommission (STIKO), Deutschland, Stand: Juli 2011 (Quelle: Deutsches Grünes Kreuz e.V.; http://dgk.de). 
tis (FSME), Gelbfieber und Tollwut. Zu den Indikationsimpfungen gehören aber auch die Impfungen von Menschen, die eine Infektion auf besonders gefährdete Personen übertragen könnten. Eine solche Indikationsimpfung wäre beispielsweise die Impfung gegen Influenza bei Krankenhauspersonal mit Patientenkontakt, bei Angehörigen immunsupprimierter Patienten oder Angehörigen von jungen Säuglingen (<6 Monate).

In der Schweiz wurde im Jahr 2006 eine dritte Kategorie, die ergänzende Impfung, definiert. Mit diesem Begriff bezeichnet man Impfungen, die aufgrund ihrer guten Schutzwirkung allen Menschen angeboten werden sollten, für die aber nicht unbedingt eine hohe Durchimpfungsrate angestrebt wird. Hierzu gehört z. B. die Pneumokokkenimpfung bei Säuglingen.

\section{Voraussetzungen für die Empfehlung eines Impfstoffs}

Die wichtigsten Voraussetzungen für die Empfehlung eines Impfstoffs sind seine Wirksamkeit, seine Sicherheit und der Nachweis der Kosten-Nutzen Effizienz. Daneben sollen noch zusätzliche Aspekte berücksichtigt werden, wie etwa die Möglichkeit zur Umsetzung der Empfehlung und die Möglichkeit eines gerechten Zugangs zur Impfung für alle Personen mit entsprechender Impfindikation. Die Impfwirksamkeit sollte auf Populationsebene überwacht werden können, und es sollte die Möglichkeit bestehen, unerwartete Nebenwirkungen frühzeitig zu erkennen (Pharmakovigilanz). Solche unerwünschten Nebenwirkungen in Zusammenhang mit einer Impfung sind meldepflichtig.

Die Wirksamkeit einer Impfung ergibt sich aus dem Anteil der Geimpften, der gegen die Infektion geschützt ist, im Vergleich zum Anteil nicht geimpfter, geschützter Personen. Sie wird nach der folgenden Formel berechnet:

$$
\text { Wirksamkeit in } \%=\frac{N \text { geimpft und infiziert } / N \text { geimpft }}{N \text { nicht geimpft und infiziert } / N \text { nicht geimpft }} \cdot 100
$$

Von Impfversagen spricht man, wenn eine Infektion trotz Impfung aufgetreten ist. Primäres Impfversagen kann auf einer ungenügenden Stimulation des Immunsystems beruhen. Sekundäres Impfversagen entsteht in der Regel durch das Absinken der Schutzwirkung im Laufe der Zeit.

Bei der Verabreichung einer Impfung müssen folgende Punkte berücksichtigt werden:

- Indikationsstellung entsprechend der offiziellen Empfehlung

- Verwendung eines Impfschemas entsprechend der offiziellen Empfehlung

- Empfohlene Verabreichungsart (z. B. intramuskulär, subkutan, Berücksichtigung der anatomischen Lokalisation)

- Berücksichtigung von Kontraindikationen (z. B. keine Verwendung von Lebendimpfstoffen bei Schwangeren oder immunsupprimierten Personen, Berücksichtigung bekannter Unverträglichkeiten)

- Beachtung der Lagerungsbedingungen (Beispiel: Einhaltung der Kühlkette)

Die Kosten für die offiziell empfohlenen Impfungen werden in der Schweiz und in Deutschland in der Regel (aber nicht zwingend) von den Krankenversicherungen über- 
nommen. Impfungen, die wegen besonderer beruflicher Exposition indiziert sind, werden in der Schweiz in der Regel vom Arbeitgeber bezahlt.

\section{Der Einfluss von Impfungen auf die Übertragungsdynamik}

Das Ziel jeder aktiven Impfung ist es, eine möglichst langdauernde, individuelle Schutzwirkung zu erzeugen. Mit steigender Durchimpfungsrate, d. h. steigendem Anteil an geimpften Personen in einer Population, kann sich eine Impfung damit auch auf die Übertragungsdynamik des Infektionserreger in der Population auswirken. Man spricht dann von einer Herdenimmunität (vgl. auch Kap. 8.1.1). Die Voraussetzungen dafür sind, dass ein Infektionserreger sein Reservoir nur im Menschen hat, und dass die Impfung nicht nur gegen die Infektion schützt, sondern auch die Übertragung verhindert. Es darf also keine nennenswerte Replikation des Erregers im geimpften Wirt stattfinden. Die sich daraus ergebende verzögerte Erregerzirkulation kann folgende Auswirkungen auf die Epidemiologie des Infektionserregers haben:

- Anstieg des durchschnittlichen Infektionsalters: Dies kann unangenehme Folgen für die Betroffenen haben, wenn der Schweregrad einer Infektion mit dem Alter zunimmt. Beobachtet wurde ein solcher Anstieg des durchschnittlichen Infektionsalters bei einigen „Kinderkrankheiten" wie Windpocken (Varizellen) und Röteln. Darüber hinaus stellt die Rötelnembryopathie als Folge einer Infektion der Mutter in der Frühschwangerschaft eine ernsthafte Gefahr für das Ungeborene dar. Weiterhin können mit Keuchhusten (Pertussis) infizierte Erwachsene zur Ansteckungsquelle für junge Säuglinge werden, die aufgrund ihres Alters noch über keinerlei Schutz verfügen und deshalb besonders gefährdet sind.

- In regelmäßigen Abständen wiederkehrende Epidemien: Dieses „Auffüllen des Pools" von empfänglichen Personen lässt sich z. B. bei Masern beobachten. Die ungenügende Durchimpfung der Bevölkerung führt u.a. in der Schweiz und in Deutschland immer wieder zu größeren Epidemien.

Wichtig ist daher, in der Bevölkerung eine möglichst hohe Übereinstimmung mit den Impfzielen (Compliance) zu erreichen. Der Verzicht auf einen Impfplan, z.B. wegen möglicher Impfkomplikationen, wäre hier ein falscher Lösungsansatz. Sowohl die Anzahl an Infizierten wie auch die Krankheitslast wären ohne einen solchen Impfplan immer höher als mit einer Impfstrategie. Die persönliche Entscheidung für oder gegen eine Impfung hat damit immer auch epidemiologische Auswirkungen, die weit über den individuellen Effekt hinausgehen.

\section{Eradikation des Infektionserregers bei genügend hoher Durchimpfungsrate}

Sinkt die Übertragungsrate unter eine kritische Schwelle, so wird der Infektionserreger entsprechend den mathematischen Modellen zur Übertragungsdynamik aussterben. Die Höhe der kritischen Schwelle $\boldsymbol{P}_{\mathbf{0}}$, bei der dies eintritt, hängt von verschiedenen Parametern wie der Kontagiosität des Erregers, der Populationsdichte und der Populationsdurchmischung ab.

Ein Beispiel für eine solche Eradikation ist das Ausrotten der Pocken. Hierfür wurde die erforderliche $P_{0}$ d. h. die Durchimpfungsrate in der Weltbevölkerung, die nötig ist, 
um die Pocken auszurotten, auf $80 \%$ geschätzt. Tatsächlich konnten die Pocken mit dieser Strategie weitgehend eliminiert werden. Nur in einigen Ländern Afrikas kam es weiterhin zu neuen Fällen. Durch eine intensivierte Impfkampagne gelang es, auch hier die Zahl der Pockenfälle einzuschränken. Die letzte natürlich erworbene Pockeinfektion wurde in Somalia am 26. Oktober 1977 diagnostiziert.

Das außerordentlich hohe Potential von Impfungen wird immer wieder durch die fehlende oder unvollständige Umsetzung von Impfzielen gefährdet. So kam es z.B. 1991 nach der Auflösung der Sowjetunion durch die nachfolgenden politischen Wirren zu einer Vernachlässigung des Impfprogramms, was eine Diphtherieepidemie zur Folge hatte.

Wie oben geschildert, tragen Impfgegner in der Schweiz und in Deutschland maßgeblich dazu bei, dass die Durchimpfungsrate bei Masern immer noch ungenügend hoch ist (<95\%), was die Erreichung des von der WHO angestrebten Ziels der Maserneradikation bislang unmöglicht macht.

\subsubsection{Chemoprophylaxe}

Unter einer Chemoprophylaxe versteht man die Gabe einer antimikrobiellen Substanz mit der Absicht, eine Infektionskrankheit zu verhindern. Diese Strategie spielt vor allem bei der Prävention von Infektionskrankheiten eine wichtige Rolle. Sie wird aber auch in anderen Bereichen der Medizin angewendet, so z.B. zur Prävention von HerzKreislauf-Erkrankungen mit Hilfe von Acetylsalicylsäure. Man unterscheidet dabei die primäre Prophylaxe, die das Auftreten einer Krankheit zu verhindern sucht, von der sekundären Prophylaxe, welche das Rezidiv einer Krankheit verhindern soll. So erhalten etwa immunsupprimierte HIV-infizierte PatientInnen eine sekundäre Prophylaxe zur Prävention opportunistischer Infektionen. Als tertiäre Prophylaxe bezeichnet man Maßnahmen, die mögliche Komplikationen einer Infektionskrankheit verhindern sollen. In diesem Kapitel werden wir auf einige Möglichkeiten der primären Prophylaxe eingehen.

\section{Perioperative Antibiotikaprophylaxe}

Die perioperative Antibiotikaprophylaxe (PAP) ist ein wichtiger Pfeiler der Prävention von chirurgischen Wundinfektionen. Das Prinzip der PAP beruht auf der Gabe von Antibiotika kurz vor dem chirurgischen Eingriff mit dem Ziel, die bakterielle Kontamination des Wundgebietes zu senken und damit das Infektionsrisiko zu verringern. Die Nachteile einer PAP sind mögliche Medikamentennebenwirkungen, die Selektion von resistenten Keimen sowie die zusätzlich entstehenden Kosten. Deshalb wird die PAP auf Eingriffe beschränkt, bei denen die Kosten-Nutzen-Effizienz erwiesen oder wahrscheinlich ist. Dies sind v.a. Eingriffe, bei denen es zu einer Verletzung von Schleimhäuten kommt (z.B. bei der Colonchirurgie) und Eingriffe, bei denen Fremdkörper implantiert werden, die im Körper verbleiben sollen (z. B. Gelenkendoprothesen). Die korrekte Verabreichung der PAP gilt als wichtiger Qualitätsparameter.

\section{Endokarditisprophylaxe}

Bei der Endokarditisprophylaxe (EP) werden ähnlich wie bei der perioperativen Prophylaxe kurz vor einem chirurgischen Eingriff Antibiotika mit dem Ziel verabreicht, das 
Risiko einer vorübergehenden Bakteriämie zu senken. Ein solches zeitweiliges Vorhandensein von Bakterien im Blut kann nachfolgend zu einer Entzündung der Herzinnenhaut (Endokarditis) führen. Eine EP wird daher bei PatientInnen mit einem erhöhten Endokarditisrisiko durchgeführt, wenn die Gefahr einer Bakteriämie bei einem Eingriff besonders hoch ist (z. B. bei operativen Schleimhautverletzungen). Ein erhöhtes Endokarditisrisiko haben z.B. PatientInnen mit einer vorbestehenden Klappenschädigung oder einer vorangegangenen Endokarditis. Aufgrund fehlender Kosten-Nutzen-Evidenz werden die Empfehlungen zur EP zunehmend enger ausgelegt.

\section{Prophylaxe von opportunistischen Infektionen}

Immunsupprimierte PatientInnen haben in Abhängigkeit vom Ausprägungsgrad der Immunsuppression ein signifikant erhöhtes Risiko, an opportunistischen Infektionen zu erkranken. Opportunistische Infektionen sind Infektionen, die durch Keime verursacht werden, die normalerweise in und auf gesunden Menschen leben, dort aber keine Krankheitserscheinungen hervorrufen. So haben beispielsweise HIV-infizierte PatientInnen mit einer geringen Zahl an CD4-Helferzellen und PatientInnen nach einer Organstransplantation eine hohes Risiko, an einer opportunistischen Infektion zu erkranken. Beispiele für einige wichtige, opportunistische Infektionen sind neben der zerebralen Toxoplasmose auch Lungenentzündungen (Pneumonien), die durch Pneumocystis jirovecii (PCP) oder Schimmelpilze hervorgerufen wurden, sowie Infektionen mit Mykobakterien oder dem Zytomegalievirus. Bei bestimmten Patientengruppen und Infektionsarten ist die medikamentöse Prophylaxe opportunistischer Infektionen inzwischen zum Standard geworden. So wird bei HIV-Infizierten mit einer CD4-Helferzellzahl von $<200 / \mathrm{mm}^{3}$ z. B. eine PCP-Prophylaxe mit Cotrimoxazol durchgeführt. Auch die WHO empfiehlt die Durchführung einer solchen Prophylaxe bei allen symptomatischen und immunsupprimierten HIV-PatientInnen. Seither hat sich diese Form der medikamentösen Prophylaxe auch in den so genannten Entwicklungsländern rasch verbreiten. In Ostafrika ließ sich die Mortalität bei HIV-PatientInnen in den ersten drei Monaten nach Beginn der anti-retroviralen Therapie (ART) dadurch bereits um 50\% senken. Dieser Effekt beruht auch auf der zusätzlichen, präventiven Wirkung des Cotrimoxazols, das den Ausbruch anderer lebensbedrohlicher Infektionen wie der zerebralen Toxoplasmose, von bakteriellen Pneumonien und Malaria verhindert.

\section{Malariaprophylaxe (siehe Kap. 8.3.4)}

\section{Postexpositionelle Prophylaxe}

Aufgabe einer postexpositionellen Prophylaxe (PEP) ist es, nach einer mutmaßlichen Ansteckung, eine Infektion bereits im latenten Stadium zu bekämpfen. Wichtige Beispiele hierfür sind die postexpositionelle Prophylaxe zur Verhütung einer HIV-Infektion, einer invasiven Meningokokkeninfektion und einer aktiven Infektion durch Mycobacterium tuberculosis. Auf diese Weise kann z. B. das Risiko einer HIV-Ansteckung nach Kontakt mit Blut oder anderen Körperflüssigkeiten und -sekreten durch die frühzeitige Einnahme einer HIV-PEP signifikant reduziert werden. Dieses Prinzip spielt u.a. eine wichtige Rolle bei der Verhütung von nosokomial erworbenen HIV-Infektionen durch Stich- oder Schnittverletzung bei Krankenhauspersonal. Auch vertikal übertragene 
Infektionen von infizierten Müttern auf ihre Kinder und Infektionen nach Vergewaltigungen oder ungeschütztem Sexualkontakt mit einer (mutmaßlich) HIV-infizierten Kontaktperson können auf diese Weise weitgehend verhindert werden. Um eine möglichst hohe Schutzwirkung zu erhalten, muss mit der HIV-PEP jedoch so früh wie möglich nach Eintritt der Risikosituation (spätestens nach 72 Stunden) begonnen werden. Zur Verhütung einer Mutter-Kind-Übertragung wird während der Schwangerschaft bei der Mutter eine konsequente antiretrovirale Therapie (ART) durchgeführt. Unmittelbar nach der Entbindung wird dann mit einer HIV-PEP beim Kind begonnen. Dank dieser Maßnahmen konnte die Zahl an vertikalen HIV-Übertragungen in den industrialisierten Ländern inzwischen drastisch gesenkt werden. Da die HIV-PEP mit einer Kombination aus verschiedenen antiretroviralen Substanzen durchgeführt wird, die zum Teil beträchtliche Nebenwirkungen haben, sollte die Indikation für eine solche Prophylaxemaßnahme immer entsprechend den geltenden Empfehlungen gestellt werden. Dies beinhaltet unter anderem eine sorgfältige Analyse der Risikosituation einschließlich der Abklärung, ob ein erhöhtes Risiko für eine Ansteckung mit Hepatitis B- und Hepatitis C-Viren sowie mit anderen sexuell übertragbaren Erregern besteht.

\subsubsection{Expositionsprophylaxe}

\section{Standardhygienemaßnahmen im Krankenhaus}

- Händedesinfektion: Die wichtigste Präventionsmaßnahme im Krankenhaus ist die hygienische Händedesinfektion. Die von der WHO festgelegten Indikationen für eine Händedesinfektion sind in Tab. 8.5 aufgelistet. Hierbei wird zwischen der direkten und der erweiterten Patientenumgebung unterschieden. Zur direkten Patientenumgebung gehören der Patient im Bett, der Nachttisch und patientenbezogene Geräte, die sich in der unmittelbaren Umgebung des Patienten befinden. Als erweiterte Patientenumgebung bezeichnet man alle darüber hinaus gehenden Bereiche des Patientenzimmers.

- Händewaschen: Die hygienische Händedesinfektion mit einem alkoholischen Händedesinfektionsmittel ist dem Händewaschen mit Wasser und Seife vorzuziehen, da sie schneller geht und wirksamer ist.

- Handschuhe: Handschuhe sollten immer dann benutzt werden, wenn es zu einem Kontakt mit Blut, Körperflüssigkeiten, Schleimhäuten oder nicht intakter Haut sowie potentiell infektiösem Material kommen kann. Darüber hinaus gibt es kaum Hinweise, dass das regelmäßige Tragen von Handschuhen im Vergleich zur regelmäßigen Händedesinfektion die Infektionsprävention verbessert. Eine Ausnahme bildet der Kontakt mit PatientInnen, die an Clostridium difficile assoziierter Diarrhoe (CDAD) leiden. Das Tragen von Handschuhen ersetzt nicht die Notwendigkeit zur Händedesinfektion!

- Mund-Nasen-Schutz: Ein normaler chirurgischer Mund-Nasen-Schutz empfiehlt sich bei PatientInnen mit einer Tröpfcheninfektion (z. B. Pertussis, Mumps, Röteln, Meningokokken-Infektion oder Angina tonsillaris, hervorgerufen durch Streptokokken der A-Gruppe), wenn die Distanz zum Patienten weniger als $1 \mathrm{~m}$ beträgt. Eine sog. FFP2oder N95-Maske sollte getragen werden, wenn bei einem Patienten eine aerogen übertragbare Infektion (z. B. Tuberkulose, Masern, Varizellen) vorliegt. 
Tab. 8.5: Indikationsgruppen nach dem WHO-Modell „Die fünf Indikationen der Händedesinfektion".

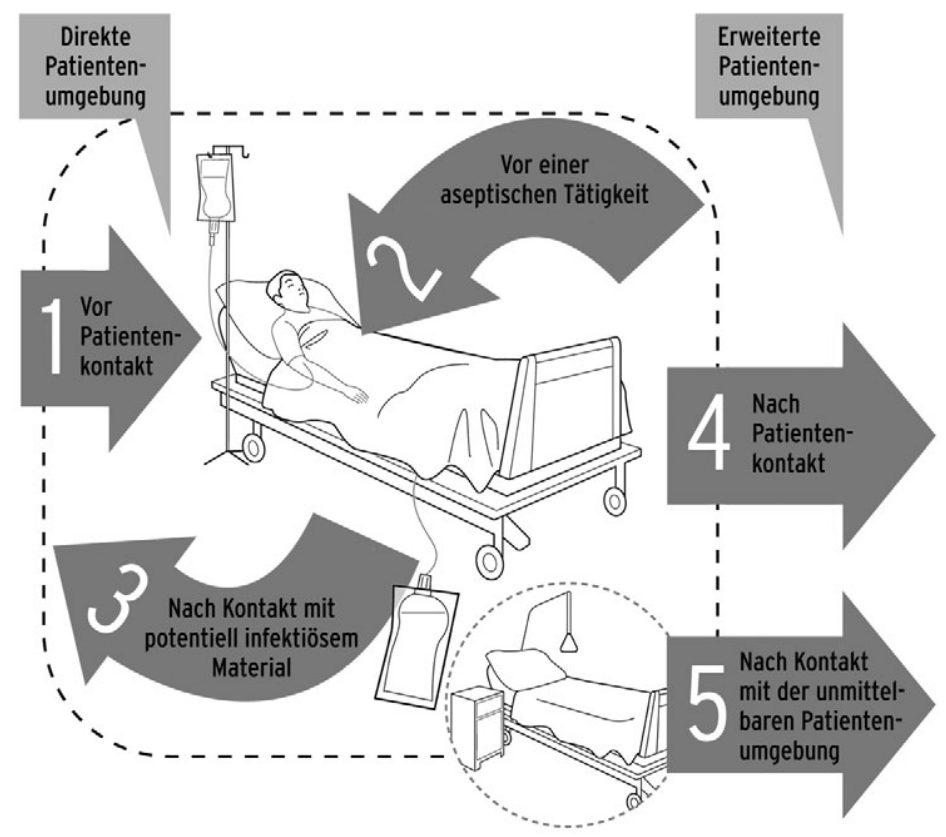

Indikationsgruppe

1. Vor Patientenkontakt

2. Vor aseptischen Tätigkeiten

3. Nach Kontakt mit potenziell infektiösen Materialien

4. Nach Patientenkontakt

5. Nach Kontakt mit der unmittelbaren Patientenumgebung
Risiko

Übertragung von Mikroorganismen von den Händen des Personals aus der erweiterten Patientenumgebung auf den Patienten

Übertragung von Mikroorganismen von den Händen des Personals in oder auf primär sterile oder nicht besiedelte Bereiche des Patienten (z. B. bei Umgang mit Kathetern, Beatmungszubehör, Schleimhäuten)

Exposition der Hände des Personals gegenüber Körperflüssigkeiten des Patienten, die potenziell infektiös sind

Übertragung von Mikroorganismen der Patientenflora auf andere Oberflächen und Patienten im Krankenhaus

Übertragung von Mikroorganismen der Patientenflora auf andere Oberflächen und Patienten im Krankenhaus

Quelle der Abbildung: „AKTION Saubere Hände“ ASH 2008-2013

- Schutzkleidung: Die normale Arbeitskleidung (= Bereichskleidung) dient v.a. dem eigenen Schutz vor einer Kontamination mit Blut und Körperflüssigkeiten. Sie soll täglich sowie bei sichtbarer Verschmutzung gewechselt werden. Zusätzlich empfohlen wird das Tragen einer Schutzkleidung (= Schutzkittel oder Überschürze) bei 
Manipulationen mit infektiösem Material sowie bei physischem Kontakt mit multiresistenten Erregern und mit Patienten, die an Infektionskrankheiten leiden. Die Schutzkleidung ist patientenbezogen zu verwenden, d.h. sie muss nach dem Kontakt mit dem Patienten gewechselt werden. Sofern ein Patient nicht isoliert wird und kein direkter Patientenkontakt gegeben ist, ist es nicht erforderlich, dass Besucher spezielle Überkittel tragen.

\section{Standardhygienemaßnahmen in der ambulanten Praxis}

Für die ambulante Praxis gelten im Prinzip dieselben Empfehlungen zur Standardhygiene wie im Krankenhaus. Da es hier keine eigene Patientenumgebung gibt, sind Händedesinfektionsmaßnahmen regelmäßig zwischen zwei Patientenkontakten erforderlich. Darüber hinaus muss eine Händedesinfektion vor aseptischen Tätigkeiten (Indikation 2, Tab. 8.4.1) und nach Kontakt mit potenziell infektiösen Materialien (Indikation 3, Tab. 8.4.1) durchgeführt werden. Eine Ausnahme bilden Praxen, in denen invasive Maßnahmen wie z. B. Hämodialysen durchgeführt werden. Dort ist das Vorgehen analog zum Prozedere im Krankenhaus.

\section{Hygienemaßnahmen in der Bevölkerung}

Außerhalb von Krankenhäusern und Praxen reicht zur Händehygiene in der Regel das Händewaschen aus. Es sollte regelmäßig vor den Mahlzeiten und nach dem Toilettengang durchgeführt werden, sowie nach Kontakt mit schmutzigen und potentiell kontaminierten Gegenständen. Ebenso sollte die "respiratorische Etikette" beachtet werden. Das bedeutet, dass bei jedem Husten oder Niesen ein Taschentuch benutzt wird. Anschließend sollten die Hände gewaschen werden. Alternativ dazu wird empfohlen, in die Ellenbeuge zu husten oder zu niesen. Wichtig ist auch die Beachtung der Lebensmittelhygiene in der Küche. Hierzu gehört z. B. das gründliche Abwaschen von potentiell kontaminierten Lebensmitteln. Nach jedem Arbeitsgang sollten die Hände gewaschen und die benutzen Gerätschaften gereinigt werden, oder es sollten neue Werkzeuge verwendet werden.

\section{Isolationsmaßnahmen}

Bei manchen Infektionskrankheiten werden zusätzlich zu den Standardmaßnahmen Isolationsmaßnahmen empfohlen (Tab. 8.6). Die zu ergreifenden Maßnahmen unterscheiden sich nach der Art der Erregerausbreitung (s. Kap. 8.3.1)

Besondere Isolierungsmaßnahmen werden bei so genannten allgemeingefährlichen Seuchen wie Pocken, Lungenpest, Lungenmilzbrand und virusbedingtem hämorrhagischem Fieber ergriffen, bei denen die Patienten vorzugsweise in speziell dafür eingerichteten Zentren behandelt werden sollen. In Deutschland sind dies v. a. spezielle Kompetenzzentren in Berlin, Hamburg, Frankfurt, München und Leipzig. Dort gibt es nicht nur einzelne Zimmer, die mit Unterdruck ausgestattet sind, sondern ein abgestuftes System von Schleusen, Vorbereitungs- und Dekontaminationsräumen sowie speziell dafür trainiertes Personal, sodass die Patienten unter diesen Bedingungen sicher behandelt werden können.

Neben diesen Isolierungsmaßnahmen zur Behandlung von infizierten oder kolonisierten Patienten gibt es noch die so genannte protektive Isolierung oder Umkehrisolie- 
Tab. 8.6: Übersicht über verschiedenen Isolationsmaßnahmen, die sich nach Art der Erregerausbreitung unterschieden.

\begin{tabular}{|c|c|c|}
\hline Erregerausbreitung & Maßnahmen & Beispiele \\
\hline Kontakt & $\begin{array}{l}\text { Vorzugsweise Einzelzimmer; } \\
\text { wenn Einzelzimmer nicht möglich, } \\
\text { dann Kohortenisolierung im Mehrbett- } \\
\text { zimmer; wenn das auch nicht möglich, } \\
\text { „Kittel-Handschuh-Pflege“ im Mehr- } \\
\text { bettzimmer }\end{array}$ & $\begin{array}{l}\text { Infektiöse Durchfallerkrankun- } \\
\text { gen, C.difficile-Infektion, } \\
\text { multiresistente Erreger }\end{array}$ \\
\hline Tröpfcheninfektion & $\begin{array}{l}\text { Vorzugsweise Einzelzimmer; } \\
\text { wenn Einzelzimmer nicht möglich, } \\
\text { Kohortenisolierung im Mehrbettzim- } \\
\text { mer; wenn das auch nicht möglich, } \\
\text { "Kittel-Handschuh- und Mund-Nasen- } \\
\text { schutz-Pflege" im Mehrbettzimmer }\end{array}$ & $\begin{array}{l}\text { Meningokokken-Infekte, } \\
\text { Pneumokokken-Infekte, } \\
\text { Pertussis, Diphtherie, Influenza, } \\
\text { Mumps, Röteln }\end{array}$ \\
\hline Luftgetragen & $\begin{array}{l}\text { Einzelzimmer, möglichst mit Unter- } \\
\text { druck; Kittel, Handschuhe, FFP2-Maske }\end{array}$ & $\begin{array}{l}\text { Tuberkulose } \\
\text { Masern, Varizellen }\end{array}$ \\
\hline
\end{tabular}

rung. Sie wird bei Patienten mit ausgeprägter Immunsuppression empfohlen. Dies sind z.B. Patienten nach Stammzelltransplantationen. Im Unterschied zur Unterbringung von Patienten in Einzelzimmern mit Unterdruck wird hier mit Überdruck gearbeitet, um das Eindringen von pathogenen Erregern in das Zimmer zu verhindern.

\section{Populationsbezogene Maßnahmen}

- Quarantäne: Die Quarantäne ist die befristete Isolierung von Personen, die verdächtig sind, an einer bestimmten Infektionskrankheit erkrankt zu sein oder Überträger dieser Krankheit zu sein. Die Zeitdauer der Quarantäne richtet sich nach der Inkubationszeit der vermuteten Krankheit. Quarantänebestimmungen gelten für die so genannten allgemeingefährlichen Seuchen (s. o.).

- Social distancing: Unter diesem Begriff fasst man Maßnahmen wie vorübergehende Schulschließungen, Verzicht auf Massenveranstaltungen etc. zusammen. Sie haben vor allem bei der Prävention einer weiteren Ausbreitung der pandemischen Influenza Bedeutung erlangt. Darüber hinaus können sie aber auch bei neu auftretenden Infektionen von großer Wichtigkeit sein (wie z. B. bei SARS). Die Art der Maßnahmen richtet sich nach dem sozialen Verhalten der vermuteten Risikogruppen.

\section{Internet-Ressourcen}

Auf unserer Lehrbuch-Homepage (www.public-health-kompakt.de) finden Sie Links zu weiterführender Literatur sowie zu anderen themenrelevanten Internet-Ressourcen. 
